\title{
Epitranscriptomic Dysregulation in Stress-induced Psychopathologies
}

\author{
Dan Ohtan Wang ${ }^{1,2}$, Kandarp Joshi ${ }^{1}$, Anand Gururajan ${ }^{3,4,5}$ \\ ${ }^{1}$ Institute for Integrated Cell-Material Sciences (iCeMS), Kyoto University, Kyoto, Japan \\ ${ }^{2}$ Wuya College of Innovation, Shenyang Pharmaceutical University, Shenyang, China. \\ ${ }^{3}$ Lambert Initiative for Cannabinoid Therapeutics, The University of Sydney, New South \\ Wales, Australia \\ ${ }^{4}$ Brain \& Mind Centre, The University of Sydney, New South Wales, Australia \\ ${ }^{5}$ School of Psychology, Faculty of Science, The University of Sydney, New South Wales, \\ Australia
}

*Corresponding Author Email: anand.gururajan@sydney.edu.au

Address: Level 6, Building F, 94 Mallett Street, Camperdown NSW 2050, Australia

Keywords: stress, epitranscriptome, transcriptome, depression, post-traumatic stress disorder 


\begin{abstract}
To date, over 100 different chemical modifications to RNA have been identified. Collectively known as the epitranscriptome, these modifications function to regulate RNA stability and as such, represent another mechanistic layer of post-transcriptional gene regulation. N6methyladenosine (m6A) is the most common RNA modification in the mammalian brain and has been implicated in a number of processes relevant to neurodevelopment, brain function and behaviour. Here, following brief descriptions on epitranscriptomic mechanisms, we will review the literature on the potential functions of the m6A-methylome in fine-tuning gene expression which include prescribing localisation of transcripts in distal compartments as well as interactions with microRNAs and long non-coding RNAs. We will then discuss findings from rodent and human studies for stress-induced disorders - major depression and post-traumatic stress disorder - which support a hypothesis for a dysregulation of the m6Amethylome and the m6A-machinery in the pathophysiology. To support this, we have included a bioinformatic analysis of publicly available single-cell RNA-sequencing and bulk transcriptomics datasets which suggests an altered m6A-methylome as a consequence of dysregulated cell- and regionally-specific expression of key enzymes involved in the 'writing, reading and erasing' of m6A. We hope this review will generate further interest in the field of epitranscriptomics, opening up new lines of research into its involvement in psychiatric disorders.
\end{abstract}




\section{Introduction}

Over recent decades, more than 100 different types of RNA chemical modifications have been identified that confer RNA transcripts with information beyond the sequence of four canonical nucleotide bases, expanding the genetic vocabulary to collectively form a new layer of post-transcriptional regulation, known as the epitranscriptome. Epitranscriptomic modifications are added during or after transcription, are generally transient, site-specific, transcript-specific, and reversible which gives them the ability to modulate gene expression in a stimulus-dependent and spatiotemporal manner ${ }^{1,2}$.

One of the most prevalent and highly specific epitranscriptomic modifications in the mammalian nervous system is N6-methyladenosine (m6A) ${ }^{3}$. A complex assortment of nuclear and cytoplasmic enzymes is involved in the 'writing' and 'erasing' of m6A at motifs which are mainly localised to 3'UTRs near stop codons of thousands of mRNAs ${ }^{4,5}$. Recent work has highlighted that the m6A-methylome in the human and mouse brain is highly specific in comparison to non-brain tissue and there are nearly 4,000 m6A-containing orthologous transcripts present in the cerebellum of both species ${ }^{3}$. As with all other RNA modifications, the m6A-methylome represents a fundamental layer of regulation in the biology of the neuron such that its absence or mutation can have a significant impact on viability ${ }^{6}$. Accordingly, over the last decade the m6A-methylome has been implicated in regulating gene expression programs involved in neurodevelopment, neuroplasticity, neurophysiology and behaviour ${ }^{7}$.

In this review, we have firstly outlined our current understanding of the m6A enzymatic machinery - 'writers, readers and erasers' - and the m6A-methylome. We then examined the m6A-directed regulation of local protein translation in various intraneuronal compartments as well as interactions between the m6A-methylome/machinery and other post-transcriptional regulatory mechanisms to fine-tune gene expression. Stress can trigger immediate effects on neurotransmission, synaptic plasticity and metabolism as well as long-lasting molecular and structural changes in the brain which are underpinned by genome-wide alterations in gene expression ${ }^{8}$. Since stress is also a major risk factor for psychiatric disorders such as major depression and post-traumatic stress disorder (PTSD), we then reviewed studies with animal models for aspects of these disorders as well as human studies which leads to our hypothesis 
that an altered m6A-methylome and the dysregulated expression of m6A-machinery is a feature of their pathophysiology.

\section{Writing, Reading \& Erasing}

\section{m6A Writers}

The methyltransferase complex (MTC) is comprised of two core proteins - the catalytic METTL3 (methyltransferase 3) and the RNA-stabilizing METTL14 (methyltransferase 14) together with several cofactor proteins including the scaffolding protein WTAP (Wilms tumor-1 associated protein), VIRMA (Protein virilizer homolog) and RBM15/15B (RNA binding protein) that facilitate identification of target modification sites and co-transcriptional m6A methylation as well as localisation of the MTC in nuclear speckles 5, 9, 10. Two other m6A writers include METTL16 and ZCCHC5 (retrotransposon gag-like protein 3) are not part of the canonical MTC and appear to target unique RNA subtypes ${ }^{11,12}$. As mentioned above, the MTC deposits $\mathrm{m} 6 \mathrm{~A}$ at a sequence motif of $\mathrm{DRACH}(\mathrm{D}=\mathrm{A} / \mathrm{G} / \mathrm{U}, \mathrm{R}=\mathrm{A} / \mathrm{G}$, $\mathrm{H}=\mathrm{A} / \mathrm{C} / \mathrm{U})$. Notably, m6A deposition at the 3'UTR is the basis for potential interactions with microRNAs that target the 3'UTR and are themselves implicated in regulating gene expression ${ }^{3,13}$. Recent studies have also shown in mammalian cells that adjacent m6A sites were prone to clustering within a 200nt region; this is evidence in support of m6A modifications interacting with each other to influence post-transcriptional processing of the same transcript ${ }^{14,15}$. While m6A deposition on RNAs is mainly a nuclear process, recent work suggests that methyltransferase activities may also occur outside the nucleus where they may take on additional functions beyond 'writing, 16-19.

Factors which determine site-specific methylation at the DRACH motif include key structural features on mRNAs such as the terminal exon-exon junction near the stop codon ${ }^{20}$, direct recruitment of the MTC to RNA Pol II ${ }^{21}$ as well as binding sites for RBM15/RBM15B located near the m6A sites ${ }^{22}$. A more critical role for METTL14 in directing site-specific methylation was identified via its interactions with the epigenetic modification, histone 3 trimethylation at Lys36 (H3K36me3). Specifically, the METTL14-H3K36me3 complex was found to direct the binding of the MTC to adjacent RNA Pol II and to deposit m6A cotranscriptionally on nascent RNAs ${ }^{23}$. Interestingly, the METTL3/METTL14 complex was also found to be involved in demethylation of histone 3 lysine 9 dimethylation (H3K9me2), via interactions with the m6A 'reader' YTHDF1 and KDM3B (lysine-specific demethylase 
3B) ${ }^{24}$. These findings would suggest that complex epigenetic-epitranscriptomic interactions exert their influence on gene expression across transcriptional and post-transcriptional processes. Transcript-specific methylation may be mediated by microRNAs as well as transcription factors which interact directly with the MTC ${ }^{25}$.

Knockout of Mettl3 has been shown to be embryonic lethal ${ }^{26}$ and conditional male and female knockouts of Mettl3 had deficits in spermatogenesis and oogenesis, respectively ${ }^{27}$. Conditional knockout of Mettl3 in the developing mouse brain caused cerebellar defects ${ }^{18}$. Conditional knockout in adult forebrain excitatory neurons was not found to have an impact on gross brain morphology, locomotor activity or anxiety-like behaviours but affected memory formation and increased marble burying behaviours ${ }^{28,29}$. Several METTL3 inhibitor compounds have been developed but their in vivo profiles are undefined ${ }^{30}$. CRISPR-Cas9 mediated knockout of Mettll4 in mice induced embryonic lethality ${ }^{31}$ and conditional knockout in the brain induced postnatal lethality ${ }^{32}$. Analysis of neural precursor cells from these conditional knockouts showed a reduction in nuclear export of m6A-RNAs ${ }^{33}$. Conditional deletion of Mettll4 from dopaminergic neurons in the mouse striatum increased neuronal excitability and impaired learning and performance ${ }^{34}$.

\section{m6A Readers}

The detection and processing of m6A-RNAs is mediated by one of two processes. Direct 'reading' involves binding of specific RNA binding proteins (RBP) whereas indirect 'reading' involves alterations to the RNA structure, thereby rendering it accessible to a different set of RBPs. The YTH domain-containing family of proteins (YTHDC1/2, YTHDF1/2/3) are direct m6A 'readers' by YTH domain binding to m6A sites ${ }^{5}$. 'Readers' also contain low-complexity domains which facilitates their phase separation into compartments such as P-bodies or neuronal granules, particularly when bound to polymethylated m6A-RNAs, creating a ready-to-process pool of transcripts ${ }^{35-37}$.

There has been considerable debate on whether each of the m6A 'readers' have unique functions or whether there is redundancy in 'reader' function. Until recently, YTHDF1 and YTHDF2 were thought to facilitate translation and degradation, respectively, and YTHDF3 was implicated in both with each DF ortholog having affinity for subsets of m6A-RNAs 5,38 , 39. It now appears, at least in specific contexts, that all three degrade m6A-RNAs ${ }^{27,} 40$. Furthermore, while depletion of individual DF proteins has minimal impact on transcript 
abundance and stability, depletion of all three leads to a stable m6A-RNA profile, suggesting that each ortholog has the ability to compensate for the loss of another but is also dependent on its unique expression profile ${ }^{40}$. So, if YTHDF1 and YTHDF3 are not involved in facilitating translation, are there any other RBP mechanisms that mediate this process? One may involve direct binding of 5'UTR m6A to the eukaryotic initiation factor 3 to initiate translation (eIF3), even in the absence of the eukaryotic initiation factor $4 \mathrm{E}$ which is a capbinding protein that normally recruits eIF3 ${ }^{41}$. Interestingly, a second mechanism may be with METTL3 itself acting as a translational enhancer. Once the MTC has methylated its target transcript, METTL3 does not disengage but rather stays bound to the RNA when it has been exported to the cytoplasm, where it then interacts with eIF3 to initiate translation ${ }^{42}$.

In mice, YTHDC1 and YTHDC2 are reportedly necessary for fertility and embryogenesis ${ }^{43-}$ 46. CRISPR-Cas9 mediated knockout of Ythdf1 in mice resulted in impairments in behaviour and hippocampal synaptic plasticity ${ }^{47}$. CRISPR-Cas9 mediated knockout of Ythdf1 or Ythdf3 in mice was not found to affect fertility or viability but knockout of Ythdf2 severely affected both these functions; Ythdf2 heterozygote mice required a functional copy of Ythdf1 or Ythdf3 to survive ${ }^{27,48}$.

A key feature of some m6A 'readers' is their reported ability to rapidly respond to changing stimuli by moving between compartments. For example, studies have shown that YTHDF1/2 are able to redistribute themselves to the nucleus or cytosol to mount an effective response to heat shock stress or viral infection ${ }^{49,50}$. The activity of neuronal YTHDF1 in particular appears to be stimulus-dependent with low basal constitutive activity but enhanced translational activity following a depolarising stimulus or in response to injury signals 47,51 .

Indirect readers bind to m6A-RNAs which have undergone a change in conformation, also known as an 'm6A-structural switch.' This change is due to the instability of the m6A.U base pair compared to A.U which results in simple, linear conformations instead of complex structures, making them more accessible to RBPs such as the nuclear fragile $\mathrm{X}$ mental retardation protein (FMRP) which can compete with YTHDF2 for binding and necessary for m6A-RNA transport into the cytoplasm ${ }^{33,52}$.

It remains unclear if m6A 'readers' show any specificity towards particular m6A-sites or m6A-RNAs. They may show preference to m6A sites on certain regions of RNAs (eg. CDS 
vs 3'UTR), interact with other RBPs such as FMRP that share similar or overlapping consensus sites or their enrichment in structures such as nuclear speckles predisposes their activity towards specific m6A- modified transcripts ${ }^{38}$. One important caveat to this discussion of direct vs indirect readers is the challenge in differentiating between the binding of an RBP directly to an m6A-RNA or binding to an m6A-RNA due to an m6A-structural switch. RNAs differ not just by methylation status but also by structure and so in interrogating the functional biology of specific readers, it would be necessary to develop sensitive methods to detect RNA structures in vivo.

Interestingly, there also appears to exist a class of so-called m6A 'anti-readers'. These proteins are repelled from the m6A-RNA by the modification, and possibly in combination with the context RNA sequence. Two such 'anti-readers' are G3BP1 and LIN28A and by binding to the same site, may counteract the destabilising effects of 'readers' on m6A-RNAs 53,54 .

m6A Erasers

Fat mass and obesity associated (FTO) protein and AlkB Homolog 5, RNA Demethylase (ALKBH5) are the two main demethylases or m6A 'erasers.' FTO is enriched in the brain and known to localise both in the nucleus and the cytoplasm; its shuttling between these two compartments is reportedly facilitated by exportin $2^{55}$. The specificity of FTO remains equivocal as evidence suggests that it preferentially demethylates another RNA modification, N6,2'-O-dimethyladenosine (m6Am), over m6A and its absence does not significantly impact m6A stoichiometries ${ }^{28,56,57}$, findings which are odds with more recent work ${ }^{58,59}$. A number of global Fto knockout mice have been generated using different gene editing strategies resulting in a variety of phenotypes. One strategy led to Fto knockout mice displaying reduced anxiety and depressive-like behaviours ${ }^{60}$, whereas another produced mice that had a hyperactive hypothalamic pituitary adrenal (HPA) axis, increased anxiety-like behaviours and impaired working memory ${ }^{61,62}$. The latter knockouts also suffered from deficiencies in the processing of brain derived neurotrophic factor (BDNF). Conditional deletion of Fto in the brain impaired growth and increased metabolism ${ }^{63}$; conditional deletion in adult excitatory neurons reduced marble burying behaviours ${ }^{28}$. Meclofenamic acid is an FTO inhibitor and a potential tool compound to further probe its functional role ${ }^{64}$. A more specific inhibitor, FB23-2, has been developed but its neuropharmacology remains unexplored $^{65}$. 
ALKBH5 is an m6A 'eraser,' colocalising with nuclear speckles, enriched in reproductive tissue ${ }^{66}$ but also found in the brain ${ }^{67}$. In vitro work has shown that a deficiency in ALKBH5 results in increased mRNA localisation in the cytoplasm in contrast to nuclear accumulation in cells with ALKBH5 suggesting that, as a consequence of its demethylating properties, ALKBH5 also regulates mRNA export into the cytoplasm ${ }^{66}$. Interestingly, recent work by Huang et al., ${ }^{68}$ has shown that localisation of ALKBH5 in astrocytes in vivo is under the regulation of circular RNA, circSTAG1 (see below). Not unlike FTO, ALKBH5 also shows promiscuity in its demethylating effects on other modifications including $\mathrm{m}_{2} \mathrm{~A}$ in ribosomal RNA ${ }^{69}$. Knockout of Alkbh5 in male mice resulted in smaller testes and defective spermatogenesis ${ }^{66,70}$.

The localisation and molecular functions of the above described 'writers,' 'readers' and 'erasers' have been represented in Figure 1. In table 1 we have described the reported phenotypes of relevant transgenic mice. 
Table 1. Genetically engineered mice to study the roles of m6A 'Writers,' 'Readers' and 'Erasers.

\begin{tabular}{|c|c|c|}
\hline Genotype & Notable phenotypes & Reference \\
\hline Mettl $^{\text {floxflox }} ;$ Nestin-Cre & $\begin{array}{l}\text { Reduced body weight at birth. } \\
\text { High mortality without artificial feeding. } \\
\text { Cerebellar hypoplasia. } \\
\text { Reduced brain size, larger ventricles. }\end{array}$ & (Wang et al., 2018) \\
\hline${\text { Mettl } 3^{\text {floxfflox }} ; \text { Nex-CreERT2 }}$ & $\begin{array}{l}\text { Increased marble burying behaviours. } \\
\text { Increased conditioned fear memory, impaired } \\
\text { extinction. }\end{array}$ & (Engel et al., 2018) \\
\hline Mettl $^{\text {floxfflox }}$; CamKIIa-Cre & $\begin{array}{l}\text { Decreased long-term memory formation; } \\
\text { compensated for by adequate training. } \\
\text { Impaired long-term potentiation. }\end{array}$ & (Zhang et al., 2018) \\
\hline $\begin{array}{l}\text { Mettl14 } 4^{\text {floxflox }} ; \operatorname{Tg}(\text { Drdl-cre }) \\
\text { Mettl14 } 4^{\text {floxflox }} ; \operatorname{Tg}(\text { Adora2a- } \\
\text { cre })\end{array}$ & $\begin{array}{l}\text { Increased neuronal excitability } \\
\text { Impaired learning and performance }\end{array}$ & (Koranda et al., 2018) \\
\hline Ythdf1 CRISPR-Cas9 KO & Impaired spatial learning and memory. & (Shi et al., 2018) \\
\hline \multirow{3}{*}{ Fto $\mathrm{KO}$} & $\begin{array}{l}\text { Decreased anxiety-like and depressive-like } \\
\text { behaviours. } \\
\text { Reduced body weight. }\end{array}$ & (Sun et al., 2019b) \\
\hline & $\begin{array}{l}\text { Stunted growth. } \\
\text { Susceptible to diet-induced obesity and higher } \\
\text { metabolism. }\end{array}$ & (Gao et al., 2010) \\
\hline & $\begin{array}{l}\text { Stunted growth. } \\
\text { Hypothalamic pituitary adrenal axis hyperactivity } \\
\text { Increased anxiety-like behaviour } \\
\text { Impaired working memory } \\
\text { Defective BDNF processing }\end{array}$ & $\begin{array}{l}\text { (Spychala \& Rüther, } \\
\text { 2019) }\end{array}$ \\
\hline Fto $^{\text {floxfflox }} ;$ Nestin-Cre & $\begin{array}{l}\text { Stunted growth. } \\
\text { Higher metabolism. }\end{array}$ & (Gao et al., 2010) \\
\hline Fto $^{\text {floxflox }} ;$ Nex-CreERT2 & $\begin{array}{l}\text { Decreased marble-burying behaviours. } \\
\text { Increased conditioned fear memory, impaired } \\
\text { extinction. }\end{array}$ & (Engel et al., 2018) \\
\hline
\end{tabular}




\section{The m6A-Methylome as a Regulator of Intraneuronal Localisation}

Gene expression in neurons is highly restricted by space and time ${ }^{71}$. As such the subcellular pre-localisation of mRNAs to axons and dendrites not only saves energy but also ensures 'ondemand' translation coupled to activity-dependent signalling pathways, in a stimulus-, transcript-specific and spatially restricted manner ${ }^{72,73}$. In this regard, m6A may travel with newly transcribed mRNAs or even direct their transport and serve as an epitranscriptomicmarks for local translation ${ }^{74}$. Indeed, we have previously identified a composite of 4,469 m6A sites distributed to 2,921 genes across the pre- and post-synaptic compartments as well as the surround glial terminals ${ }^{17}$. There were 1,266 hypermethylated synaptic genes which were enriched for a number of different functions such as synapse assembly, maturation, organisation, modulation of transmission, whereas the hypomethylated synaptic genes are enriched for metabolic functions. This functionally differential methylation for synaptic genes further supports a potential role of m6A in tagging transcripts for activity-dependent translation/decay that may be mediated by the expression of 'readers' 17, 33 .

Additionally, m6A machinery may also be localised to specific subregions. As mentioned above, Fto mRNA can be locally translated in axons in response to stimuli such as the nerve growth factor to demethylate Gap43 mRNA which leads to axonal elongation and axon growth ${ }^{75}$. Similarly, axonal YTHDF1 has a role in facilitating the translation of m6AmRNAs in crossing spinal commissural neurons and its deletion has been shown to result in the mis-projection of pre-crossing axons into motor columns ${ }^{76}$.

Taken together, the accumulating evidence indicates an important role of $\mathrm{m} 6 \mathrm{~A}$ in maintaining the 'regional autonomy' and functionality of distal, non-nuclear compartments in the form of 'ready-to-process' pools of m6A-transcripts as well as the m6A machinery that are perhaps critical for energy-efficient, 'on-demand' responses to stimuli such as stress ${ }^{77}$. The precise operational mechanisms via which transcripts are actually transported is unknown but could involve phase separation ${ }^{35-37}$. 


\section{Noncoding RNA interactions with the m6A-methylome}

\section{MicroRNAs}

MicroRNAs (miRNAs) are small (21-23nt) non-coding RNAs but with a significant role in RNA silencing pathways including mRNA degradation, translational repression and transcriptional silencing ${ }^{78}$. They are abundantly expressed in the brain where they have been implicated in a number of critical processes including neurodevelopment, synaptic plasticity as well as in the pathophysiology of major depression and PTSD ${ }^{79}$. MiRNAs are derived from primary microRNAs (pri-miRNAs). Evidence has shown that m6A marks these primiRNAs for DGCR8-mediated cleavage into the mature miRNAs through m6A reader, HNRNPA2B1 ${ }^{80}$. Depletion of METTL3 in vitro reduced the binding of DGCR8 to primiRNAs and resulted in a global reduction of mature miRNAs and concomitant accumulation of unprocessed pri-miRNAs ${ }^{81}$. Even though mature microRNAs are short in length, recent evidence has shown that they too may harbour m6A in their sequence ${ }^{82}$.

There is accumulating evidence for interactions between microRNAs and the m6Amethylome beginning with observations by Meyer et al. ${ }^{13}$ who reported that $67 \%$ of 3'UTRs with m6A sites in mammalian RNAs contain at least one microRNA prediction target. Shortly after, Chen et al. reported that (a) deposition of m6A on target RNAs is dependent on microRNAs, (b) microRNAs are able to induce de novo m6A-methylation and (c) microRNA expression can influence nuclear localisation of METTL3 ${ }^{83}$. Moreover, by virtue of its ability to regulate RNA splicing, m6A can also regulate the length of 3'UTRs and thus the availability of miRNA targets ${ }^{20}$.

Transcripts for 'writers', 'readers' and 'erasers' are themselves targets of microRNAs. For example, miR-145 was found to negatively regulate the expression of YTHDF2 in hepatocellular carcinoma tissue ${ }^{84}$. To further explore the potential interactions between microRNAs and the m6A machinery, we used the microRNA database miRNet ${ }^{85}$ and identified 132 unique microRNAs which are able to regulate expression of METTL3, METTL14, YTHDC1, YTHDC2, YTHDF1, YTHDF2, YTHDF3, FTO and ALKBH5 in the human brain (Supplementary Table 1). As can be seen in the network plot (Figure 2), some of these microRNAs are presumably able to regulate expression of more than one of these targets which is consistent with their promiscuous mechanism of action. 


\section{Long Non-Coding}

Long non-coding RNAs (lncRNAs) are longer (>200nt) non-coding RNAs. Their synthesis profile has some features that are very similar to mRNAs, including splicing and the formation of secondary structures. Amongst their many functions as post-transcriptional regulators of gene expression, they can act as precursors to microRNAs, regulate their function by acting as decoys, compete with binding sites with mRNAs or by forming complexes with microRNAs ${ }^{86-88}$. LncRNAs have been implicated in the pathophysiology of depression and PTSD ${ }^{89-91}$.

The mechanistic evidence for interaction between lncRNAs and m6A machinery is scarce with only study so far showing that the lncRNA FOXM1-AS facilitates the interaction between FOXM1 mRNA and ALKBH5 which leads to increased expression of FOXM1 ${ }^{92}$. However, there are many more studies which have reported on the impact of epitranscriptomic modifications including m6A on lncRNA function ${ }^{93}$. For example, m6Amethylation of the lncRNA metastasis associated lung adenocarcinoma transcript 1 (MALAT1) was found to influence the physical access (see above "m6A-structural switch) to RNA-binding proteins ${ }^{94}$; m6A-methylation of the lncRNA X-inactive specific transcript (XIST) and its subsequent recognition by YTHDC1 was a precursor to transcriptional silencing ${ }^{22}$. While long intergenic non-coding RNAs (lincRNAs) are also known to harbour $\mathrm{m} 6 \mathrm{~A}^{95}$, evidence for the functional role of m6A in lincRNAs is limited except for one study which showed that m6A-methylation of linc1281 was necessary for differentiation of mouse embryonic stem cells ${ }^{96}$. 


\section{Stress-induced Psychopathologies and the m6A-Methylome}

The neuroendocrine response to stress, also known as the HPA axis, involves the initial release of corticotropin releasing factor (CRF) by the paraventricular nucleus of the hypothalamus and amygdala, which stimulates CRF receptors (CRFR) on the anterior pituitary to release adrenocorticotropic hormone (ACTH), ultimately resulting in the release of corticosteroids by the adrenal cortex. Corticosteroids can bind to gluco- (GR) and mineralocorticoid receptors (MR) on a range of target organs including the brain to initiate fast non-genomic effects (e.g. glutamate release, endocannabinoid release) as well as slower genomics effects ${ }^{97,98}$.

In this section, we have firstly reviewed evidence from animal studies which demonstrate the impact of stress on the m6A-methylome/machinery and its relationship to stress-induced behavioural changes of relevance to major depression and PTSD (Table 2). Stressors can be either acute or chronic but the difference in impact is rather diffuse ${ }^{98}$. For the discussion below on major depression, we have focused on models which utilise restraint stress or chronic unpredictable stress. For the discussion on PTSD, we have chosen models which use variations of the fear conditioning paradigm. We then examined human studies which report evidence for a dysregulated m6A-methyome/machinery in these two disorders, incorporating a bioinformatic meta-analysis of publicly available datasets (Supplementary Table 2). 
Table 2. Summary of preclinical studies which have used stress paradigms to assess impact on m6A profile in various brain regions.

\begin{tabular}{|c|c|c|c|c|c|c|}
\hline Reference & Wigdado et al., (2016) & Walters et al., (2017) & Engel et al., (2018) & Zhang et al., (2018) & Huang et al., (2020b) & Song et al., (2020) \\
\hline $\begin{array}{l}\text { Mouse } \\
\text { strain }\end{array}$ & $\mathrm{C} 57 \mathrm{BL} / 6$ & $\begin{array}{ll}\text { C57BL/6NTac } & \mathrm{x} \\
\text { 129S6/SvEvTac } & \\
\end{array}$ & C57BL/6 & C57BL/6J & $\mathrm{C} 57 \mathrm{BL} / 6 \mathrm{~J}$ & C57BL/6J \\
\hline Age, Sex & Adult, male & Adult, male & Adult, male & Adult, male & Adult, male & Adult, unknown \\
\hline Paradigm & $\begin{array}{l}\text { Cued-fear } \\
\text { conditioning - CS; } \\
2 \mathrm{~min}, 80 \mathrm{~dB}+\mathrm{US} ; 1 \mathrm{~s}, \\
0.7 \mathrm{~mA})\end{array}$ & $\begin{array}{l}\text { Contextual fear } \\
\text { conditioning - US; } 3 \mathrm{x} \\
0.5 \mathrm{~mA}, 1 \mathrm{~min} \text { ITI }\end{array}$ & 15 min restraint stress & $\begin{array}{l}\text { Contextual fear } \\
\text { conditioning - US; } \\
0.8 \mathrm{~mA}, 2 \mathrm{~s}\end{array}$ & $\begin{array}{l}\text { Chronic unpredictable } \\
\text { stress for } 4 \text { weeks }\end{array}$ & $\begin{array}{l}\text { Chronic unpredictable } \\
\text { stress for } 4 \text { weeks }\end{array}$ \\
\hline $\begin{array}{l}\text { Region of } \\
\text { Interest }\end{array}$ & $\mathrm{mPFC}$ & Dorsal HIP & PFC and AMG & HIP & HIP & VTA \\
\hline $\begin{array}{l}\text { m6A } \\
\text { analysis }\end{array}$ & MeRIP-seq & EpiQuik m6A ELISA & $\begin{array}{l}\text { EpiQuik m6A ELISA } \\
\text { LC-MS/MS }\end{array}$ & miCLIP-m6A-seq & $\begin{array}{l}\text { EpiQuik m6A ELISA } \\
\text { MeRIP-seq }\end{array}$ & $\begin{array}{l}\text { Epitranscriptomic } \\
\text { Microarray }\end{array}$ \\
\hline $\begin{array}{l}\text { Summary } \\
\text { of findings }\end{array}$ & $\begin{array}{l}\text { Increased m6A } 2 \mathrm{~h} \\
\text { after fear } \\
\text { conditioning. } \\
\text { Knockdown of Fto in } \\
\text { the mPFC increased } \\
\text { consolidation of cued } \\
\text { fear. }\end{array}$ & $\begin{array}{l}\text { Increased m6A } 0.5 \mathrm{~h} \\
\text { after fear } \\
\text { conditioning. } \\
\text { Knockdown or } \\
\text { knockout of Fto } \\
\text { increased } \\
\text { consolidation of } \\
\text { contextual fear. }\end{array}$ & $\begin{array}{l}\text { Increased m6A in the } \\
\text { prefrontal cortex, } \\
\text { decreased levels in the } \\
\text { amygdala. }\end{array}$ & $\begin{array}{l}\text { Samples analysed at } \\
\text { various time points } \\
\text { revealed highly } \\
\text { dynamic m6A profile. } \\
\text { But } 1183 \text { genes were } \\
\text { m6A modified at all } \\
\text { time points, enriched } \\
\text { for synaptic plasticity } \\
\text { and neural } \\
\text { development. }\end{array}$ & $\begin{array}{l}\text { Decreased m6A, } \\
\text { reversed by } \\
\text { circSTAG1 over- } \\
\text { expression. }\end{array}$ & $\begin{array}{l}\text { CUS increased m6A } \\
\text { which was reduced by } \\
\text { deep brain stimulation } \\
\text { for a proportion of genes, } \\
\text { concomitant with } \\
\text { attenuated CUS- } \\
\text { behavioural phenotypes. }\end{array}$ \\
\hline
\end{tabular}




\section{Major Depression - Animal Studies}

In rodents, many strategies exist to induce aspects of depression, which includes exposure to a restraint or chronic unpredictable stress (CUS) paradigm. Engel et al. ${ }^{28}$ analysed epitranscriptomic changes $4 \mathrm{~h}$ after 15 mins of restraint stress with mouse cortical RNA samples using m6A-seq. They showed at baseline that half of all expressed cortical genes were m6A methylated and most of these were implicated in synaptic plasticity and neuronal regulation. However, $4 \mathrm{~h}$ after restraint stress, the magnitude of differential gene expression compared to controls was low and furthermore, the impact on cortical m6A levels was also insignificant; the authors speculate that these findings were likely to be due to heterogenous nature of input material which may have precluded the identification of regional effects of the restraint stress. Based on these results, the authors focused on examining the epitranscriptome on total RNA from the prefrontal cortex (PFC) and the amygdala (AMG). At 30 mins after the stress, there were no differences in global m6A profiles. But from $1 \mathrm{~h}$ to $24 \mathrm{~h}$ the m6A levels were higher in the AMG and lower in the PFC; these results were confirmed for up to $4 \mathrm{hrs}$ using isolated mRNA and LC-MS. This tissue-specific result was matched by lower mRNA expression of Fto, Alkbh5 and Ythdcl in the AMG than in the PFC over the same time period. The impact of acute restraint stress on global m6A/m levels was also detected in the blood along with altered mRNA expression of Mettl3, Wtap and Alkbh5. The authors then applied m6A-RIP-qPCR to show differences in the absolute methylation of a number of specific stress and synaptic plasticity-related transcripts in the PFC and AMG. Interestingly, they reported a negative correlation between stress-induced changes in gene expression and absolute $\mathrm{m} 6 \mathrm{~A} / \mathrm{m}$ levels which led to the suggestion that these modifications were mainly linked to transcript decay ${ }^{28}$.

Huang et al., ${ }^{68}$ reported a CUS-induced reduction in hippocampal m6A levels and m6A-seq revealed a greater down-regulation in m6A-methylation than up-regulation of a number of transcripts, including for fatty acid amide hydrolase (Faah) mRNA. CUS also induced an increase in hippocampal (HIP) FAAH protein expression. This enzyme is a major component of the endocannabinoid system which metabolises the archetypal endocannabinoid, anandamide (AEA) ${ }^{99}$ and is also critical to the stress response ${ }^{100}$. Sustained FAAH activity have been associated with maladaptive stress responses ${ }^{101}$. The authors also reported that there was CUS-induced localisation of ALKBH5 in the nucleus of astrocytes whereas under control conditions, ALKBH5 is normally bound to the circular RNA, circSTAG1, in the cytoplasm. The expression of $\operatorname{circSTAG1}$ in the HIP, blood and plasma of CUS mice was 
lower than controls and this profile was matched by similar differential expression in the blood and plasma in patients with major depression. Overexpression of circSTAG1 captured and restricted ALKBH5 to the cytoplasm, preventing the demethylation of nascent Faah mRNA molecules, degradation in the cytoplasm and the attenuation of depressive-like behaviours. It is worth highlighting that other transcripts of interest with significantly decreased m6A-methylation include Nape-pld, an enzyme involved in the synthesis of endocannabinoids, Fkbp5 as well as Alkbh5 ${ }^{68}$.

Another recent study also used the CUS paradigm in mice to examine the impact on the m6A-methylome in the ventral tegmental area (VTA), another stress-sensitive brain structure involved in the reward system ${ }^{102}$. In contrast with the previous study, CUS-induced a significant increase in m6A-methylation of transcripts in the VTA. Deep brain stimulation (DBS), which is reportedly an effective form of treatment for major depression, reversed CUS-induced depressive-like behaviours as well as changes in m6A-methylation status for 329 genes. Notably, there were also a number of genes for which the expression was not altered between CUS and CUS-DBS treated mice, but the m6A-methylation status was significantly different. A correlation analysis also revealed no significant relationship between m6A-methylation status and gene expression.

\section{Major Depression - Human Studies}

To date, there has been only one study to quantify m6A levels in patients suffering from major depression. Engel et al., ${ }^{28}$ first administered oral dexamethasone to healthy controls and observed a reduction in m6A/m levels, mRNA expression of METTL3, FTO, ALKBH5, YTHDF1, YTHDF2 and an increase in YTHDF3 in the blood. They then administered a dexamethasone challenge test to healthy controls and patients, as a way of assessing glucocorticoid receptor resistance ${ }^{103}$, and observed a reduction in global $\mathrm{m} 6 \mathrm{~A} / \mathrm{m}$ in blood only in the controls. These results were confirmed by similarly treating B lymphocyte cell lines (BLCLs) derived from controls and patients with dexamethasone or cortisol. They then performed m6A-seq on control and patient BLCLs treated with cortisol, observing that there were more cortisol-regulated m6A peaks in BLCLs from controls than patients. Additionally, m6A-RIP-qPCR revealed a significant decrease in absolute $\mathrm{m} 6 \mathrm{~A} / \mathrm{m}$ for several stress response genes, including FKBP5, following cortisol treatment in BCLs from controls but not patients. FKBP5 is a chaperone protein which modulates activity of the glucocorticoid receptor and has been implicated in stress-induced psychopathologies ${ }^{104}$. In the absence of 
differences in expression of the glucocorticoid receptor in the blood, these results led to the conclusion that signalling processes downstream of receptor activation are likely to be altered in patients, affecting the methylation status of stress response transcripts such as FKBP5 ${ }^{28}$.

A number of studies have identified single nucleotide polymorphisms (SNP) in the FTO gene which appears to moderate a complex relationship between obesity and major depression ${ }^{105-}$ 108 . At least one of these SNPs (rs9939609) has a limited association with major depression 109. A SNP in ALKBH5 gene has also been associated with depression ${ }^{110}$.

To further explore the potential role for a dysregulated m6A-methylome in major depression, we identified and analysed relevant publicly available single-nucleus (sn) and bulk RNA-seq datasets from the NCBI GEO database (see Supplementary Info for Methods). We hypothesized that the features of the pathophysiology of depression may include altered expression of $\mathrm{m} 6 \mathrm{a}$ 'writers, readers and erasers'.

The snRNA-seq study consisted of gene expression profiles of $\sim 70000$ cells derived from prefrontal cortex (PFC) of post-mortem brain samples of 17 patients diagnosed with major depression who had died due to suicide and 17 matched healthy controls who died due to accidents or natural causes ${ }^{111}$. Heatmap of mean log normalised expression for m6A machinery genes in different PFC cell subtypes is represented in Figure 3. Genes encoding 'writer' METTL16, 'readers' YTHDC1 and YTHDC2 (marked by yellow boxes), show significant differential expression in three neuronal cell types. Specifically, METTL16 was upregulated in both L5/6 excitatory pyramidal neurons and inhibitory somatostatin neurons in patients. YTHDC1 and YTHDC2 were down- and upregulated respectively, in inhibitory somatostatin neurons.

Bulk transcriptomics datasets from several other brain regions and noted significant differential expression of 'writers,' 'readers,' and 'erasers' was detected in examined structures except for PFC (Figure 4).

Given the influence of the m6A-methylome on gene expression, we next investigated if there was a relationship between the number of m6A sites per gene and differential gene expression in major depression. For this, we used an m6A-seq dataset generated from human 
cerebrum and cerebellum and exomePeak to determine the number of m6A sites in each gene 3,112. Using the bulk transcriptomics datasets above, we then grouped differentially expressed genes in patients vs controls based on the m6A peaks count in respective brain regions. $\log 2$ fold change $(\log 2 \mathrm{FC})$ values (patients vs controls) for genes were presented in cumulative distribution function plots (Figure 5). The analysis showed sex-specific effects as well as effects between and within brain regions. These have been briefly discussed below. The findings from other analyses have been discussed in the Supplementary Information (Supplementary Figure 1).

The relationship between differential gene expression and the number of m6A sites per gene was highly dynamic in females compared to males across and within brain regions (Figure 5A-F). For example, in the study by Chang et al., ${ }^{113}$, in the BA9 region of dorsolateral prefrontal cortex (DLPFC) female samples, there was a larger distribution of genes with one m6A site that were downregulated. Similarly in the BA25 region of anterior cingulate cortex (ACC) female samples, there was a larger distribution of genes with more than five m6A sites which showed downregulation as represented by the higher cumulative distribution of genes for $\log 2 \mathrm{FC}$ lower than zero (Figure 5A, C). However, in matched male samples for the BA9 region of DLPFC, there was a larger distribution of genes with more than five m6A sites that were downregulated as compared to remaining genes; this was also the case in the BA25 region of ACC for males (Figure 5B, D). In another study which also analysed the DLPFC of males and females, but with no layer information, there was a larger distribution of downregulated genes with more than five m6A sites in females (Figure 5E) ${ }^{114}$. However in males, there was a uniform distribution of $\log 2 \mathrm{FC}$ values for genes with more than five m6A sites (Figure 5F).

In comparing the DLPFC and ACC, the differences in differential gene expression for genes with five or less m6A sites was comparable. However, for genes with more than five m6A sites, there was a greater proportion of upregulated genes in the ACC than in the DLPFC (Figure 5G-H) ${ }^{115}$. In comparing gene expression in L3 and L5 pyramidal neurons of DLPFC, our analysis of a third study showed that genes containing m6A sites had similar distribution of $\log 2 \mathrm{FC}$ in L3 pyramidal neurons but in L5 pyramidal neurons there was a greater distribution of genes with more than five m6A sites that were downregulated compared to remaining groups (Figure 5I-J) ${ }^{116}$. 


\section{Post-Traumatic Stress Disorder - Animal Studies}

The psychology of PTSD is rooted in the acquisition of experiences and formation of memories associated with specific traumatic events. Accordingly, persistent changes in brain structures such as the HIP and AMG with roles in learning and memory have been implicated in the pathophysiology of this disorder ${ }^{117}$. A commonly used paradigm to induce aspects of PTSD in rodents involves fear conditioning. It must be noted however that the focus of the majority of studies reviewed below was to examine the role of the epitranscriptome in the context of learning and memory with unconditioned stimuli (US) that are weaker in intensity compared to those used to induce long-lasting, robust phenotypes of relevance to PTSD in rodents ${ }^{118}$. This caveat imposes limits on the conclusions that can be drawn from the studies in the context of this disorder.

In an early study by Widagdo et al., ${ }^{119}$, a cued-fear conditioning procedure was used in which adult male mice were exposed to an auditory conditioned stimulus (CS, tone) paired with a US $(0.7 \mathrm{~mA}, 1 \mathrm{~s})$. Each mouse received 6 x CS-US pairs with an intertrial interval of 2 min. The mice were sacrificed $2 \mathrm{~h}$ later and RNA from the medial prefrontal cortex (mPFC) was analysed with m6A-seq. Results showed that compared to naïve mice, there was a significant increase in the number of m6A peaks in the CS-US mice for mPFC genes involved in synaptic plasticity and most were located within the vicinity of the stop codon. The fear conditioning paradigm caused no change in the mRNA expression of Mettl3 or Mettl14 but decrease in Fto. The authors then used a cortical neuronal culture system and m6A-IP-qPCR to validate the increased m6A levels for several of the identified plasticityrelated genes. Using this in vitro setup, they also showed that the over-expression or knocking down of FTO was able to alter the decay rate of specific transcripts. And in their final experiment, they knocked down Fto in the mPFC of mice and showed that while this manipulation had no impact on fear acquisition, it did affect memory consolidation with the mice showing increased freezing behaviour in response to the cue $24 \mathrm{~h}$ after the training ${ }^{119}$.

Another early study by Walters et al. ${ }^{120}$ used a contextual fear conditioning paradigm. Mice were placed inside the fear conditioning chamber and exposed to three $0.5 \mathrm{~mA}$ foot shocks with 1 min intervals between shocks. The mice were sacrificed 0.5 or $1 \mathrm{~h}$ later, and the HIP was used for analysis. At the $0.5 \mathrm{~h}$ time point, there was no effect in m6A levels in total RNA but a significant increase in m6A levels in mRNA. There was also a significant decrease in 
expression of FTO, mainly in the synaptosome (a tissue preparation with enriched synaptic terminals). The authors then used CRISPR-Cas9 and a knockdown approach targeting Fto in the dorsal HIP, to demonstrate that it was involved in the consolidation of contextual fear memories ${ }^{120}$.

Following on from their examination of the impact of acute restraint stress on the m6Amethylone, Engel et al., ${ }^{28}$ generated Mettl3 and Fto conditional knockout mice deficient in expression in excitatory neurons in the neocortex and HIP. They reported that while absence of Mettl3 caused a reduction in global m6A levels, conditional deletion of Fto had no effect. They also generated conditional knockout mice with a timed deletion of Mettl3 and Fto in pyramidal neurons of the dorsal and ventral HIP. The baseline behavioural phenotypes of these mice were comparable to wildtypes, except for increased and decreased marble burying behaviour in the Mettl3 and Fto conditional knockout mice, respectively. All conditional knockout mice and wildtypes were subjected to a fear conditioning procedure with a single CS $(80 \mathrm{~dB}$ tone, $20 \mathrm{~s})$ paired with a US $(0.7 \mathrm{~mA}, 2 \mathrm{~s})$ and then assessed for cued- or contextual memories. There was no difference in acquisition compared to wildtypes but both conditional knockout mice displayed increased cued- and contextual fear memory that was resistant to extinction. This phenotype was underpinned by fear-conditioning induced effects on expression of genes for neurotransmission and transcription which were more pronounced in Fto conditional knockout mice than in the Mettl3 conditional knockout mice compared to unstressed conditional knockout mice ${ }^{28}$.

Using a contextual fear conditioning paradigm, Zhang et al. ${ }^{29}$ showed that the number of m6A-modified genes in the HIP were dynamically regulated at 0.5, 1 and $4 \mathrm{~h}$ following contextual fear conditioning with a single US $(0.8 \mathrm{~mA}, 2 \mathrm{~s})$. However, there were 1183 genes that were m6A-modified at all time points and these were enriched for synaptic plasticity and neural development. These authors also showed that Mettl3 conditional knockout mice displayed reduced freezing to the context $24 \mathrm{~h}$ but not $30 \mathrm{~min}$ after training which suggested a deficit in long-term memory formation. This deficit could be corrected by restoring METTL3 expression in the dorsal HIP. However, when these conditional knockout mice were exposed to three foot shocks instead of one, contextual fear memory was no different to wildtypes. Interestingly, in comparing the temporal effects of the fear conditioning (one shock) between wildtype and Mettl3 cKO mice, there were no differentially expressed genes in the hippocampus over 4 hours. However, the absence of METTL3 led to a significant 
reduction in the number of m6A-modified transcripts for several immediate early genes. Further analysis revealed a significant reduction in the protein expression for these IEGs which led to the conclusion that METTL3 is necessary to modify and shunt m6A-IEG transcripts down a translational pathway to facilitate memory consolidation in the HIP following fear conditioning ${ }^{29}$.

To investigate the functional roles of the m6A reader YTHDF1 in learning and memory, Shi et al., ${ }^{47}$ generated CRISPR-Cas9 knockout mice. For fear conditioning, mice received either one of three different stimuli: a single pairing of a CS tone $(75 \mathrm{~dB}, 30 \mathrm{~s})$ with a US foot shock $(0.5 \mathrm{~mA}, 1 \mathrm{~s})$ for the weak protocol, a single pairing of the CS tone with a longer US $(2 \mathrm{~s})$ for the moderate protocol or three pairings of the CS tone with the longer US and an inter-trial interval of $1 \mathrm{~min}$ for the strong protocol. During the inter-trial interval with the moderate training protocol, Ythdf1 knockout mice froze less than the wildtypes but not when the tone was presented, suggesting that contextual learning was impaired in these mice. Ytdhfl knockout mice displayed deficits in contextual fear memory when assessed $24 \mathrm{~h}$ but not $2 \mathrm{~h}$ later and these deficits were rescued with the hippocampal re-expression of YTDHF1. Contextual-fear memory was also reduced in these mice $2 \mathrm{~h}$ after the weaker training protocol but there were no genotype differences with the stronger protocol at $24 \mathrm{~h}^{47}$.

\section{Post-Traumatic Stress Disorder - Human Studies}

To date, there have been no studies which have specifically profiled the m6Aepitranscriptome or expression of m6A machinery in patients with PTSD but similar to our approach above, we searched for and bioinformatically analysed publicly available datasets. We observed differential expression of m6A machinery genes in peripheral blood samples from PTSD patients compared to controls (Figure 6). There was a significant dysregulation of several 'writers' (GSE81761, GSE67663) and 'readers' (GSE81761, GSE860) but no effect on 'erasers.' Sleep treatment reduced expression of the 'writer' RBM15 and the indirect 'reader' IGF2BP2 (PTSD_Notimproved_vs_Improved, GSE81761) in patients who did not reported improvements in symptomatology. In patients who had been admitted to the emergency room (PTSD_vs_NoPTSD_ER) following trauma, there was a downregulation in YTHDC1 and YTHDF2 in peripheral blood mononuclear cells (PMBCs), but this difference was not detected 4 months later(PTSD_vs_NoPTSD_M4, GSE860). 
In a recent preprint, Jaffe et.al. ${ }^{115}$ reported differentially expressed genes in PTSD patients as compared to controls after correcting for various factors in a cohort of 107 PTSD patients. We examined differential expression of m6A machinery genes in PTSD vs controls from four brain regions (viz. two cortical regions; DLPFC and ACC and two amygdala regions; basolateral amygdala (BLA) and medial amygdala (MeA)). Expression of the m6A 'eraser' ALKBH5 was significantly upregulated in ACC and MeA regions of the brain in PTSD patients as compared to control. Similarly, we also observed that components of the MTC such as METTL3 and CBLL1 were downregulated in the ACC and upregulated in the DLPFC, respectively (Figure 6). We constructed cumulative distribution function plots for these brain regions and observed that in the DLPFC, there was a larger distribution of upregulated genes with at least one m6A site compared to genes with no m6A sites which showed a uniform distribution of up and downregulated genes. In the ACC, there was a larger distribution of downregulated genes with no m6A sites and larger distribution of upregulated genes with two or more m6A sites (Figure 7).

Overall, the evidence from animal model investigations strongly implicate a role for m6Amethylome/machinery genes in mediating aspects of the response to acute and chronic stress. Moreover, our bioinformatic analysis of publicly available human data for these disorders indicate that not only dysregulation of m6A-machinery genes is detectable in multiple brain regions and in the periphery, but altered expression of m6A-methylome is affected by the number of m6A modifications peaks on the genes. 


\section{Conclusions \& Future Directions}

The field of epitranscriptomics is rapidly evolving and largely driven by advancements in massively parallel sequencing, bioinformatics, and public databases. Despite the widespread nature of m6A modification sites, the majority are unmethylated at baseline ${ }^{121}$. The functional relevance of the constitutive m6A sites versus regulated m6A sites is unknown but such sub-stoichiometric levels of m6A would indicate a large margin in which to regulate neuronal gene expression. Furthermore, while the lifecycle of m6A modifications is thought to be dynamic, the degree of dynamicity and whether it varies from transcript to transcript is unknown. The apparent clustering of modifications on the same transcript also suggests that individual modifications are unlikely to have a significant functional effect on their own; rather they may interact with each other in as yet unknown ways to influence transcript stability and gene expression as can be seen in our cumulative distribution plot analyses. Adding to this complexity is our observation that there does not appear to be a clear correlation between the number of m6A sites and differential gene expression. Therefore, to functionally interrogate specific modification sites is challenging. Accordingly, using strategies to manipulate the epitranscriptomic machinery of 'writers', 'erasers' and 'readers' while low in resolution, is relatively easier and just as informative in the interim, until RNAmodification detection, mapping and manipulation technologies have been refined and matured. Additionally, future investigations should consider incorporating techniques such as ribosome profiling and proteomics (Buccitelli \& Selbach, 2020).

In terms of the mechanisms which link stress exposure to changes in the m6A-methylome and/or functional expression of m6A machinery, we speculate that they are likely embedded

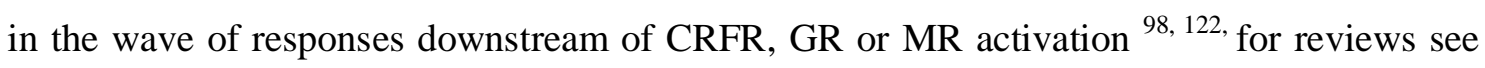
123, 124. For example, this could include the transport and 'on-demand' processing of m6ARNAs (e.g. FAAH) in distal compartments such as the synapse. Additionally, stress can induce expression of non-coding RNAs which are susceptible to m6A-methylation, thus interfering with or enhancing their ability to finetune expression of genes which may include those involved in the stress response or even which form the m6A-machinery. Taken together, this array of complex transcriptional and post-transcriptional effects may underpin, in part, changes in synaptic plasticity, neurotransmission and energy metabolism required to mount a transient, behavioural response to stress that is adaptive. Maladaptive responses may emerge if any of the above mechanisms are not efficiently terminated, leading to the onset of 
behavioural phenotypes of relevance to major depression and PTSD. In this regard, research into the role of the m6A-methylome/machinery as potential mediators of resilience to stress is an exciting prospect.

As we have shown, very few human studies have been carried out on the epitranscriptome, but our analysis of publicly available data shows sex-differences in major depression and PTSD. Future research should also consider the potential influence of individual risk factors (e.g. early-life adversity, drug abuse) on the m6A-methylome/machinery and whether these interactions influence disorder onset. It is worth highlighting that given the clinical presentation of these disorders is heterogenous and varies with time, we have yet to explore the short- and long-term impact of stress on the m6A-methylome/machinery and how they might correlate with clinically relevant phenotypes.

And finally, evidence has shown that post-transcriptional mechanisms are implicated in the mechanism of action for therapeutics for the treatment of major depression and PTSD (O'Connor et al., 2012; Murphy \& Singewald, 2018). It would be interesting to examine if m6A-methylome/machinery is similarly amenable to therapeutic targeting.

In conclusion, our review has highlighted significant findings from animal and human studies in support of a hypothesis for the involvement of the m6A-methylome/machinery in the pathophysiology of stress-induced disorders such as major depression and PTSD. Future research incorporating the experimental strategies described above will significantly advance our understanding on the impact of stress on epitranscriptomic mechanisms in the brain in both healthy and psychiatric states. These mechanisms may in turn form the basis for developing next-generation therapeutics to treat these disorders. 


\section{Acknowledgements}

DOW is supported by KAKENHI 17H03546, 19H04907, 19H05212, AMED 18dm0307023h 0001, NSFC 31971335, Xingliao Talents Program, XLYC1802007, Department of Education of Liaoning Province 1911520092, Hirose research grant, Takeda research grant. AG is supported by University of Sydney Research Fellowship.

\section{Competing Interests}

The authors declare no conflict of interest. 


\section{Figure Legends}

Figure 1 - The regulation of m6A-RNAs. The 'writer' enzymes which form the methyltransferase complex (MTC) deposit methyl groups on the adenosine nucleotides of newly transcribed RNAs. These m6A-RNAs can remain complexed with the MTC and exported out into the cytoplasm for translation, demethylated by 'eraser' enzymes such as FTO and ALKBH5, undergo splicing and export by YTHDC1 or degradation by YTHDC2. In the cytoplasm, m6A-RNAs are recognized by 'reader' proteins (e.g. YTHDF1/2/3 and YTHDC2) which mediate their degradation or translation initiation. The modified RNAs can also undergo demethylation by cytoplasmic demethylase FTO. The compartmentalised expression of 'writers, readers and erasers' is likely to be dynamically regulated; the cell can distribute and redistribute these proteins between nuclear and cytoplasmic/synaptic compartments in a context-dependent manner.

Figure 2 - Network of the microRNAs (red squares) which regulate expression of m6A machinery genes (black circles). The size of the circles reflects the number of microRNAs which are predicted to regulate expression in humans. Generated using miRNET 2.0.

Figure 3 - Heatmap of snRNA-seq mean log-normalised expression for m6A machinery genes in the PFC of major depression patients and healthy controls. The four yellow boxes highlight differentially expressed m6A 'readers', YTHDC1/2 and the m6A 'writer' METTL16 in somatostatin inhibitory neurons and excitatory neurons $(\mathrm{p}<0.05)$. Astros: astrocytes; Endo: endothelia; Ex: Excitatory neurons; Inhib: Inhibitory neurons; Micro/Macro: Microglia/Macrophage; Oligos: Oligodendrocytes; OPCs: Oligodendrocyte precursor cells.

Figure 4 - Heatmap of $\log 2 \mathrm{FC}$ for m6A machinery gene expression in bulk RNAseq/microarray expression profiling experiments in patient samples vs controls from different brain regions and conditions. Top three index bars describe the brain regions, subregions, sex of the subjects and the bottom row indicates the reference or NCBI GEO series ID. Black bars indicate either no subregion analysis or unspecified details on the sex of the subjects in the relevant studies. Grey areas in the heatmap indicate no differential gene expression. F: females; M: males; Mix: both) (ACC: anterior cingulate cortex; AMY: amygdala; CBLM: cerebellum; DLPFC: dorsolateral prefrontal cortex; FC: frontal cortex; HPC: hippocampus; 
NACC: nucleus accumbens; OVPFC: orbital ventral prefrontal cortex; PC: parietal cortex; PFC: prefrontal cortex; STR: striatum.

Figure 5 - Cumulative distributions of $\log 2 \mathrm{FC}$ of gene expression in MDD patients vs control show sex- and brain region-dependent regulation. The differentially expressed genes are divided into four groups based on the number of m6a sites mapped to their RNA. m6a_0: no m6a peaks; m6a_1: one m6a peak; m6a_2_5: two to five m6a peaks; m6a_6: six or more m6a peaks. DLPFC: dorsolateral prefrontal cortex; ACC: anterior cingulate cortex.

Figure 6 - Heatmap of $\log 2 \mathrm{FC}$ for m6A machinery gene expression in blood and brain regions of PTSD patients. Index bars above the heatmap indicates comparisons and sample source. BLA: basolateral amygdala; MeA: medial amygdala; ACC: anterior cingulate cortex; DLPFC: dorsolateral prefrontal cortex.

Figure 7 - Cumulative distribution plot for $\log 2 \mathrm{FC}$ of significant differential genes in PTSD patients as compared to controls in different conditions. The genes are divided in four groups based on the number of m6a sites mapped to their RNA. m6a_0: no m6a peaks; m6a_1: one m6a peak; m6a_2_5: two to five m6a peaks; m6a_6: six or more m6a peaks. DLPFC: dorsolateral prefrontal cortex; ACC: anterior cingulate cortex. 


\section{References}

1. Jonkhout N, Tran J, Smith MA, Schonrock N, Mattick JS, Novoa EM. The RNA modification landscape in human disease. RNA 2017; 23(12): 1754-1769.

2. Schaefer M, Kapoor U, Jantsch MF. Understanding RNA modifications: the promises and technological bottlenecks of the 'epitranscriptome'. Open Biol 2017; 7(5).

3. Liu J, Li K, Cai J, Zhang M, Zhang X, Xiong X et al. Landscape and Regulation of m(6)A and m(6)Am Methylome across Human and Mouse Tissues. Mol Cell 2020; 77(2): 426-440.e426.

4. Zhao BS, Roundtree IA, He C. Post-transcriptional gene regulation by mRNA modifications. Nat Rev Mol Cell Biol 2017; 18(1): 31-42.

5. Zaccara S, Ries RJ, Jaffrey SR. Reading, writing and erasing mRNA methylation. Nat Rev Mol Cell Biol 2019; 20(10): 608-624.

6. Destefanis E, Avşar G, Groza P, Romitelli A, Torrini S, Pir P et al. A mark of disease: how mRNA modifications shape genetic and acquired pathologies. RNA 2020.

7. Livneh I, Moshitch-Moshkovitz S, Amariglio N, Rechavi G, Dominissini D. The $\mathrm{m}(6)$ A epitranscriptome: transcriptome plasticity in brain development and function. Nat Rev Neurosci 2020; 21(1): 36-51.

8. Floriou-Servou A, von Ziegler L, Waag R, Schläppi C, Germain P-L, Bohacek J. The acute stress response in the multi-omic era. Biol Psychiatry 2021.

9. Garcias Morales D, Reyes JL. A birds'-eye view of the activity and specificity of the mRNA m6A methyltransferase complex. WIREs RNA 2020; n/a(n/a): e1618.

10. Liu L, Song B, Ma J, Song Y, Zhang S-Y, Tang Y et al. Bioinformatics approaches for deciphering the epitranscriptome: Recent progress and emerging topics. Comput Struct Biotechnol J 2020; 18: 1587-1604.

11. Pendleton KE, Chen B, Liu K, Hunter OV, Xie Y, Tu BP et al. The U6 snRNA m6A Methyltransferase METTL16 Regulates SAM Synthetase Intron Retention. Cell 2017; 169(5): 824-835.e814.

12. Ma H, Wang X, Cai J, Dai Q, Natchiar SK, Lv R et al. N6-Methyladenosine methyltransferase ZCCHC4 mediates ribosomal RNA methylation. Nat Chem Biol 2019; 15(1): 88-94.

13. Meyer Kate D, Saletore Y, Zumbo P, Elemento O, Mason Christopher E, Jaffrey Samie R. Comprehensive Analysis of mRNA Methylation Reveals Enrichment in $3^{\prime}$ UTRs and near Stop Codons. Cell 2012; 149(7): 1635-1646.

14. Meyer KD. DART-seq: an antibody-free method for global m6A detection. Nat Methods 2019; 16(12): 1275-1280. 
15. Zhang Z, Chen LQ, Zhao YL, Yang CG, Roundtree IA, Zhang Z et al. Single-base mapping of m(6)A by an antibody-independent method. Sci Adv 2019; 5(7): eaax0250.

16. Lin S, Choe J, Du P, Triboulet R, Gregory Richard I. The m6A Methyltransferase METTL3 Promotes Translation in Human Cancer Cells. Mol Cell 2016; 62(3): 335345.

17. Merkurjev D, Hong W-T, Iida K, Oomoto I, Goldie BJ, Yamaguti H et al. Synaptic N6-methyladenosine (m6A) epitranscriptome reveals functional partitioning of localized transcripts. Nat Neurosci 2018; 21(7): 1004-1014.

18. Wang C-X, Cui G-S, Liu X, Xu K, Wang M, Zhang X-X et al. METTL3-mediated m6A modification is required for cerebellar development. PLoS Biol 2018; 16(6): e2004880-e2004880.

19. Xu H, Dzhashiashvili Y, Shah A, Kunjamma RB, Weng YL, Elbaz B et al. m(6)A mRNA Methylation Is Essential for Oligodendrocyte Maturation and CNS Myelination. Neuron 2020; 105(2): 293-309.e295.

20. Ke S, Alemu EA, Mertens C, Gantman EC, Fak JJ, Mele A et al. A majority of m6A residues are in the last exons, allowing the potential for 3' UTR regulation. Genes \& development 2015; 29(19): 2037-2053.

21. Slobodin B, Han R, Calderone V, Vrielink JAFO, Loayza-Puch F, Elkon R et al. Transcription Impacts the Efficiency of mRNA Translation via Co-transcriptional N6adenosine Methylation. Cell 2017; 169(2): 326-337.e312.

22. Patil DP, Chen C-K, Pickering BF, Chow A, Jackson C, Guttman M et al. m6A RNA methylation promotes XIST-mediated transcriptional repression. Nature 2016; 537(7620): 369-373.

23. Huang $\mathrm{H}$, Weng $\mathrm{H}$, Zhou $\mathrm{K}$, Wu T, Zhao BS, Sun M et al. Histone H3 trimethylation at lysine 36 guides m6A RNA modification co-transcriptionally. Nature 2019; 567(7748): 414-419.

24. Li Y, Xia L, Tan K, Ye X, Zuo Z, Li M et al. N6-Methyladenosine cotranscriptionally directs the demethylation of histone H3K9me2. Nat Genet 2020.

25. Bertero A, Brown S, Madrigal P, Osnato A, Ortmann D, Yiangou L et al. The $\mathrm{SMAD} 2 / 3$ interactome reveals that TGF $\beta$ controls m6A mRNA methylation in pluripotency. Nature 2018; 555(7695): 256-259.

26. Geula S, Moshitch-Moshkovitz S, Dominissini D, Mansour AA, Kol N, SalmonDivon $\mathrm{M}$ et al. Stem cells. m6A mRNA methylation facilitates resolution of naïve pluripotency toward differentiation. Science (New York, NY) 2015; 347(6225): 1002 1006. 
27. Lasman L, Krupalnik V, Geula S, Zerbib M, Viukov S, Mor N et al. Contextdependent functional compensation between Ythdf m6A readers. bioRxiv 2020: 2020.2006.2003.131441.

28. Engel M, Eggert C, Kaplick PM, Eder M, Röh S, Tietze L et al. The Role of m6A/mRNA Methylation in Stress Response Regulation. Neuron 2018; 99(2): 389-403.e389.

29. Zhang Z, Wang M, Xie D, Huang Z, Zhang L, Yang Y et al. METTL3-mediated N6methyladenosine mRNA modification enhances long-term memory consolidation. Cell Res 2018; 28(11): 1050-1061.

30. Bedi RK, Huang D, Eberle SA, Wiedmer L, Śledź P, Caflisch A. Small-Molecule Inhibitors of METTL3, the Major Human Epitranscriptomic Writer. ChemMedChem 2020; 15(9): 744-748.

31. Meng T-G, Lu X, Guo L, Hou G-M, Ma X-S, Li Q-N et al. Mettl14 is required for mouse postimplantation development by facilitating epiblast maturation. FASEB $\mathrm{Jl}$ 2019; 33(1): 1179-1187.

32. Yoon K-J, Ringeling FR, Vissers C, Jacob F, Pokrass M, Jimenez-Cyrus D et al. Temporal Control of Mammalian Cortical Neurogenesis by m6A Methylation. Cell 2017; 171(4): 877-889.e817.

33. Edens BM, Vissers C, Su J, Arumugam S, Xu Z, Shi H et al. FMRP Modulates Neural Differentiation through m6A-Dependent mRNA Nuclear Export. Cell Rep 2019; 28(4): 845-854.e845.

34. Koranda JL, Dore L, Shi H, Patel MJ, Vaasjo LO, Rao MN et al. Mettl14 Is Essential for Epitranscriptomic Regulation of Striatal Function and Learning. Neuron 2018; 99(2): 283-292.e285.

35. Fu Y, Zhuang X. m6A-binding YTHDF proteins promote stress granule formation by modulating phase separation of stress granule proteins. bioRxiv 2019: 694455 .

36. Gao Y, Pei G, Li D, Li R, Shao Y, Zhang QC et al. Multivalent m6A motifs promote phase separation of YTHDF proteins. Cell Res 2019; 29(9): 767-769.

37. Ries RJ, Zaccara S, Klein P, Olarerin-George A, Namkoong S, Pickering BF et al. $\mathrm{m}(6)$ A enhances the phase separation potential of mRNA. Nature 2019; 571(7765): 424-428.

38. Shi H, Wei J, He C. Where, When, and How: Context-Dependent Functions of RNA Methylation Writers, Readers, and Erasers. Mol Cell 2019; 74(4): 640-650.

39. Rauch S, He E, Srienc M, Zhou H, Zhang Z, Dickinson BC. Programmable RNAGuided RNA Effector Proteins Built from Human Parts. Cell 2019; 178(1): 122134.e112.

40. Zaccara S, Jaffrey SR. A Unified Model for the Function of YTHDF Proteins in Regulating m6A-Modified mRNA. Cell 2020; 181(7): 1582-1595.e1518. 
41. Meyer KD, Patil DP, Zhou J, Zinoviev A, Skabkin MA, Elemento O et al. 5' UTR m(6)A Promotes Cap-Independent Translation. Cell 2015; 163(4): 999-1010.

42. Xiao W, Adhikari S, Dahal U, Chen Y-S, Hao Y-J, Sun B-F et al. Nuclear m6A Reader YTHDC1 Regulates mRNA Splicing. Mol Cell 2016; 61(4): 507-519.

43. Bailey AS, Batista PJ, Gold RS, Chen YG, de Rooij DG, Chang HY et al. The conserved RNA helicase YTHDC2 regulates the transition from proliferation to differentiation in the germline. eLife 2017; 6: e26116.

44. Hsu PJ, Zhu Y, Ma H, Guo Y, Shi X, Liu Y et al. Ythdc2 is an N6-methyladenosine binding protein that regulates mammalian spermatogenesis. Cell Res 2017; 27(9): $1115-1127$.

45. Wojtas MN, Pandey RR, Mendel M, Homolka D, Sachidanandam R, Pillai RS. Regulation of m(6)A Transcripts by the $3^{\prime} \rightarrow 5^{\prime}$ RNA Helicase YTHDC2 Is Essential for a Successful Meiotic Program in the Mammalian Germline. Mol Cell 2017; 68(2): 374-387.e312.

46. Kasowitz SD, Ma J, Anderson SJ, Leu NA, Xu Y, Gregory BD et al. Nuclear m6A reader YTHDC1 regulates alternative polyadenylation and splicing during mouse oocyte development. PLoS Genet, vol. 142018, p e1007412.

47. Shi H, Zhang X, Weng Y-L, Lu Z, Liu Y, Lu Z et al. m6A facilitates hippocampusdependent learning and memory through YTHDF1. Nature 2018; 563(7730): 249253.

48. Li M, Zhao X, Wang W, Shi H, Pan Q, Lu Z et al. Ythdf2-mediated m6A mRNA clearance modulates neural development in mice. Genome Biol 2018; 19(1): 69.

49. Zhou J, Wan J, Gao X, Zhang X, Jaffrey SR, Qian S-B. Dynamic m6A mRNA methylation directs translational control of heat shock response. Nature 2015; 526(7574): 591-594.

50. Hao H, Hao S, Chen H, Chen Z, Zhang Y, Wang J et al. N6-methyladenosine modification and METTL3 modulate enterovirus 71 replication. Nucleic Acids Res 2018; 47(1): 362-374.

51. Weng YL, Wang X, An R, Cassin J, Vissers C, Liu Y et al. Epitranscriptomic m6A Regulation of Axon Regeneration in the Adult Mammalian Nervous System. Neuron 2018; 97(2): 313-325.e316.

52. Hsu PJ, Shi H, Zhu AC, Lu Z, Miller N, Edens BM et al. The RNA-binding protein FMRP facilitates the nuclear export of N6-methyladenosine-containing mRNAs. $J$ Biol Chem 2019; 294(52): 19889-19895.

53. Edupuganti RR, Geiger S, Lindeboom RGH, Shi H, Hsu PJ, Lu Z et al. N(6)methyladenosine $(\mathrm{m}(6) \mathrm{A})$ recruits and repels proteins to regulate mRNA homeostasis. Nat Struct Mol Biol 2017; 24(10): 870-878. 
54. Sun L, Fazal FM, Li P, Broughton JP, Lee B, Tang L et al. RNA structure maps across mammalian cellular compartments. Nat Struct Mol Biol 2019; 26(4): 322-330.

55. Gulati P, Avezov E, Ma M, Antrobus R, Lehner P, O'Rahilly S et al. Fat mass and obesity-related (FTO) shuttles between the nucleus and cytoplasm. Biosci Rep 2014; 34(5): $\mathrm{e} 00144$.

56. Hess ME, Hess S, Meyer KD, Verhagen LAW, Koch L, Brönneke HS et al. The fat mass and obesity associated gene (Fto) regulates activity of the dopaminergic midbrain circuitry. Nat Neurosci 2013; 16(8): 1042-1048.

57. Mauer J, Luo X, Blanjoie A, Jiao X, Grozhik AV, Patil DP et al. Reversible methylation of m6Am in the $5^{\prime}$ cap controls mRNA stability. Nature 2017; 541(7637): 371-375.

58. Huang H, Wang Y, Kandpal M, Zhao G, Cardenas H, Ji Y et al. FTO-Dependent N6Methyladenosine Modifications Inhibit Ovarian Cancer Stem Cell Self-Renewal by Blocking cAMP Signaling. Cancer Res 2020: canres.4044.2020.

59. Kisliouk T, Rosenberg T, Ben-Nun O, Ruzal M, Meiri N. Early-life m6A RNA demethylation by fat mass and obesity-associated protein (FTO) influences resilience or vulnerability to heat stress later in life. eNeuro 2020: ENEURO.0549-0519.2020.

60. Sun L, Ma L, Zhang H, Cao Y, Wang C, Hou N et al. Fto Deficiency Reduces Anxiety- and Depression-Like Behaviors in Mice via Alterations in Gut Microbiota. Theranostics 2019; 9(3): 721-733.

61. Fischer J, Koch L, Emmerling C, Vierkotten J, Peters T, Brüning JC et al. Inactivation of the Fto gene protects from obesity. Nature 2009; 458(7240): 894-898.

62. Spychala A, Rüther U. FTO affects hippocampal function by regulation of BDNF processing. PLoS One 2019; 14(2): e0211937-e0211937.

63. Gao X, Shin Y-H, Li M, Wang F, Tong Q, Zhang P. The Fat Mass and Obesity Associated Gene FTO Functions in the Brain to Regulate Postnatal Growth in Mice. PLoS One 2010; 5(11): e14005.

64. Huang Y, Yan J, Li Q, Li J, Gong S, Zhou H et al. Meclofenamic acid selectively inhibits FTO demethylation of m6A over ALKBH5. Nucleic Acids Res 2015; 43(1): 373-384.

65. Huang Y, Su R, Sheng Y, Dong L, Dong Z, Xu H et al. Small-Molecule Targeting of Oncogenic FTO Demethylase in Acute Myeloid Leukemia. Cancer Cell 2019; 35(4): 677-691.e610.

66. Zheng G, Dahl John A, Niu Y, Fedorcsak P, Huang C-M, Li Charles J et al. ALKBH5 Is a Mammalian RNA Demethylase that Impacts RNA Metabolism and Mouse Fertility. Mol Cell 2013; 49(1): 18-29. 
67. Du T, Li G, Yang J, Ma K. RNA Demethylase Alkbh5 is Widely Expressed in Neurons and Decreased during Brain Development. Brain Res Bull 2020.

68. Huang R, Zhang Y, Bai Y, Han B, Ju M, Chen B et al. N6-Methyladenosine Modification of Fatty Acid Amide Hydrolase Messenger RNA in Circular RNA STAG1-Regulated Astrocyte Dysfunction and Depressive-like Behaviors. Biol Psychiatry 2020; 88(5): 392-404.

69. Ensfelder TT, Kurz MQ, Iwan K, Geiger S, Matheisl S, Müller M et al. ALKBH5induced demethylation of mono- and dimethylated adenosine. Chem Commun 2018; 54(62): 8591-8593.

70. Tang C, Klukovich R, Peng H, Wang Z, Yu T, Zhang Y et al. ALKBH5-dependent m6A demethylation controls splicing and stability of long 3'-UTR mRNAs in male germ cells. Proc Natl Acad Sci USA 2018; 115(2): E325.

71. Wang DO, Martin KC, Zukin RS. Spatially restricting gene expression by local translation at synapses. Trends Neurosci 2010; 33(4): 173-182.

72. Di Liegro CM, Schiera G, Di Liegro I. Regulation of mRNA transport, localization and translation in the nervous system of mammals (Review). Int J Mol Med 2014; 33(4): 747-762.

73. Holt CE, Martin KC, Schuman EM. Local translation in neurons: visualization and function. Nat Struct Mol Biol 2019; 26(7): 557-566.

74. Roy R, Shiina N, Wang DO. More dynamic, more quantitative, unexpectedly intricate: Advanced understanding on synaptic RNA localization in learning and memory. Neurobiol Learn Mem 2020; 168: 107149.

75. Yu J, Chen M, Huang H, Zhu J, Song H, Zhu J et al. Dynamic m6A modification regulates local translation of mRNA in axons. Nucleic Acids Res 2018; 46(3): 14121423.

76. Zhuang M, Li X, Zhu J, Zhang J, Niu F, Liang F et al. The m6A reader YTHDF1 regulates axon guidance through translational control of Robo3.1 expression. Nucleic Acids Res 2019; 47(9): 4765-4777.

77. Madugalle SU, Meyer K, Wang DO, Bredy TW. RNA N6-Methyladenosine and the Regulation of RNA Localization and Function in the Brain. Trends Neurosci 2020; 43(12): 1011-1023.

78. Kosik KS. The neuronal microRNA system. Nat Rev Neurosci 2006; 7(12): 911-920.

79. Issler O, Chen A. Determining the role of microRNAs in psychiatric disorders. Nat Rev Neurosci 2015; 16(4): 201-212.

80. Alarcón Claudio R, Goodarzi H, Lee H, Liu X, Tavazoie S, Tavazoie Sohail F. HNRNPA2B1 Is a Mediator of m6A-Dependent Nuclear RNA Processing Events. Cell 2015; 162(6): 1299-1308. 
81. Alarcón CR, Lee H, Goodarzi H, Halberg N, Tavazoie SF. N6-methyladenosine marks primary microRNAs for processing. Nature 2015; 519(7544): 482-485.

82. Berulava T, Rahmann S, Rademacher K, Klein-Hitpass L, Horsthemke B. N6Adenosine Methylation in MiRNAs. PLoS One 2015; 10(2): e0118438.

83. Chen T, Hao Y-J, Zhang Y, Li M-M, Wang M, Han W et al. m6A RNA Methylation Is Regulated by MicroRNAs and Promotes Reprogramming to Pluripotency. Cell Stem Cell 2015; 16(3): 289-301.

84. Yang Z, Li J, Feng G, Gao S, Wang Y, Zhang S et al. MicroRNA-145 Modulates N(6)-Methyladenosine Levels by Targeting the 3'-Untranslated mRNA Region of the N(6)-Methyladenosine Binding YTH Domain Family 2 Protein. J Biol Chem 2017; 292(9): 3614-3623.

85. Chang L, Zhou G, Soufan O, Xia J. miRNet 2.0: network-based visual analytics for miRNA functional analysis and systems biology. Nucleic Acids Res 2020; 48(W1): W244-W251.

86. Qureshi IA, Mattick JS, Mehler MF. Long non-coding RNAs in nervous system function and disease. Brain Res 2010; 1338: 20-35.

87. Ransohoff JD, Wei Y, Khavari PA. The functions and unique features of long intergenic non-coding RNA. Nat Rev Mol Cell Biol 2018; 19(3): 143-157.

88. Cuevas-Diaz Duran R, Wei H, Kim DH, Wu JQ. Invited Review: Long non-coding RNAs: important regulators in the development, function and disorders of the central nervous system. Neuropathol Appl Neurobiol 2019; 45(6): 538-556.

89. Issler O, van der Zee YY, Ramakrishnan A, Wang J, Tan C, Loh Y-HE et al. SexSpecific Role for the Long Non-coding RNA LINC00473 in Depression. Neuron 2020; 106(6): 912-926.e915.

90. Daskalakis NP, Provost AC, Hunter RG, Guffanti G. Noncoding RNAs: Stress, Glucocorticoids, and Posttraumatic Stress Disorder. Biol Psychiatry 2018; 83(10): 849-865.

91. Yoshino Y, Dwivedi Y. Non-Coding RNAs in Psychiatric Disorders and Suicidal Behavior. Front Psychiatry 2020; 11: 543893.

92. Zhang S, Zhao BS, Zhou A, Lin K, Zheng S, Lu Z et al. m(6)A Demethylase ALKBH5 Maintains Tumorigenicity of Glioblastoma Stem-like Cells by Sustaining FOXM1 Expression and Cell Proliferation Program. Cancer Cell 2017; 31(4): 591606.e596.

93. Jacob R, Zander S, Gutschner T. The Dark Side of the Epitranscriptome: Chemical Modifications in Long Non-Coding RNAs. Int J Mol Sci 2017; 18(11). 
94. Zhou KI, Parisien M, Dai Q, Liu N, Diatchenko L, Sachleben JR et al. N(6)Methyladenosine Modification in a Long Noncoding RNA Hairpin Predisposes Its Conformation to Protein Binding. J Mol Biol 2016; 428(5 Pt A): 822-833.

95. Batista PJ, Molinie B, Wang J, Qu K, Zhang J, Li L et al. m(6)A RNA modification controls cell fate transition in mammalian embryonic stem cells. Cell Stem Cell 2014; 15(6): 707-719.

96. Yang D, Qiao J, Wang G, Lan Y, Li G, Guo X et al. N6-Methyladenosine modification of lincRNA 1281 is critically required for $\mathrm{mESC}$ differentiation potential. Nucleic Acids Res 2018; 46(8): 3906-3920.

97. McEwen BS, Akil H. Revisiting the Stress Concept: Implications for Affective Disorders. J Neurosci 2020; 40(1): 12.

98. Floriou-Servou A, von Ziegler L, Waag R, Schläppi C, Germain P-L, Bohacek J. The acute stress response in the multi-omic era. Biological Psychiatry 2021.

99. Di Marzo V. New approaches and challenges to targeting the endocannabinoid system. Nat Rev Drug Discov 2018; 17(9): 623-639.

100. Morena M, Patel S, Bains JS, Hill MN. Neurobiological Interactions Between Stress and the Endocannabinoid System. Neuropsychopharmacology 2016; 41(1): 80-102.

101. Mayo LM, Asratian A, Lindé J, Holm L, Nätt D, Augier G et al. Protective effects of elevated anandamide on stress and fear-related behaviors: translational evidence from humans and mice. Mol Psychiatry 2020; 25(5): 993-1005.

102. Song N, Du J, Gao Y, Yang S. Epitranscriptome of the ventral tegmental area in a deep brain-stimulated chronic unpredictable mild stress mouse model. Transl Neurosci 2020; 11(1): 402-418.

103. Menke A, Arloth J, Pütz B, Weber P, Klengel T, Mehta D et al. Dexamethasone stimulated gene expression in peripheral blood is a sensitive marker for glucocorticoid receptor resistance in depressed patients. Neuropsychopharmacology 2012; 37(6): $1455-1464$.

104. Zannas AS, Wiechmann T, Gassen NC, Binder EB. Gene-Stress-Epigenetic Regulation of FKBP5: Clinical and Translational Implications. Neuropsychopharmacology 2016; 41(1): 261-274.

105. Kivimaki M, Jokela M, Hamer M, Geddes J, Ebmeier K, Kumari M et al. Examining overweight and obesity as risk factors for common mental disorders using fat mass and obesity-associated (FTO) genotype-instrumented analysis: The Whitehall II Study, 1985-2004. Am J Epidemiol 2011; 173(4): 421-429.

106. Rivera M, Cohen-Woods S, Kapur K, Breen G, Ng MY, Butler AW et al. Depressive disorder moderates the effect of the FTO gene on body mass index. Mol Psychiatry 2012; 17(6): 604-611. 
107. Samaan Z, Anand SS, Zhang X, Desai D, Rivera M, Pare G et al. The protective effect of the obesity-associated rs9939609 A variant in fat mass- and obesityassociated gene on depression. Mol Psychiatry 2013; 18(12): 1281-1286.

108. Rivera M, Locke AE, Corre T, Czamara D, Wolf C, Ching-Lopez A et al. Interaction between the FTO gene, body mass index and depression: meta-analysis of 13701 individuals. Br J Psychiatry 2017; 211(2): 70-76.

109. Yao Y, Wen Y, Du T, Sun N, Deng H, Ryan J et al. Meta-analysis indicates that SNP rs9939609 within FTO is not associated with major depressive disorder (MDD) in Asian population. J Affect Disord 2016; 193: 27-30.

110. Du T, Rao S, Wu L, Ye N, Liu Z, Hu H et al. An association study of the m6A genes with major depressive disorder in Chinese Han population. J Affect Disord 2015; 183: 279-286.

111. Nagy C, Maitra M, Tanti A, Suderman M, Théroux J-F, Davoli MA et al. Singlenucleus transcriptomics of the prefrontal cortex in major depressive disorder implicates oligodendrocyte precursor cells and excitatory neurons. Nat Neurosci 2020; 23(6): 771-781.

112. Meng J, Cui X, Rao MK, Chen Y, Huang Y. Exome-based analysis for RNA epigenome sequencing data. Bioinformatics 2013; 29(12): 1565-1567.

113. Chang L-C, Jamain S, Lin C-W, Rujescu D, Tseng GC, Sibille E. A Conserved BDNF, Glutamate- and GABA-Enriched Gene Module Related to Human Depression Identified by Coexpression Meta-Analysis and DNA Variant Genome-Wide Association Studies. PLoS One 2014; 9(3): e90980.

114. Hagenauer MH, Schulmann A, Li JZ, Vawter MP, Walsh DM, Thompson RC et al. Inference of cell type content from human brain transcriptomic datasets illuminates the effects of age, manner of death, dissection, and psychiatric diagnosis. PLoS One 2018; 13(7): e0200003.

115. Jaffe AE, Barry BK, Tao R, Tran MN, Page SC, Maynard KR et al. Decoding shared versus divergent transcriptomic signatures across cortico-amygdala circuitry in PTSD and depressive disorders. bioRxiv 2021: 2021.2001.2012.426438.

116. Arion D, Huo Z, Enwright JF, Corradi JP, Tseng G, Lewis DA. Transcriptome Alterations in Prefrontal Pyramidal Cells Distinguish Schizophrenia From Bipolar and Major Depressive Disorders. Biol Psychiatry 2017; 82(8): 594-600.

117. Maren S, Holmes A. Stress and Fear Extinction. Neuropsychopharmacology 2016; 41(1): 58-79.

118. Richter-Levin G, Stork O, Schmidt MV. Animal models of PTSD: a challenge to be met. Mol Psychiatry 2019; 24(8): 1135-1156. 
119. Widagdo J, Zhao Q-Y, Kempen M-J, Tan MC, Ratnu VS, Wei W et al. ExperienceDependent Accumulation of N6-Methyladenosine in the Prefrontal Cortex Is Associated with Memory Processes in Mice. J Neurosci 2016; 36(25): 6771.

120. Walters BJ, Mercaldo V, Gillon CJ, Yip M, Neve RL, Boyce FM et al. The Role of The RNA Demethylase FTO (Fat Mass and Obesity-Associated) and mRNA Methylation in Hippocampal Memory Formation. Neuropsychopharmacology 2017; 42(7): 1502-1510.

121. He PC, He C. m6A RNA methylation: from mechanisms to therapeutic potential. EMBO J 2021; n/a(n/a): e105977.

122. Mifsud KR, Reul JMHM. Mineralocorticoid and glucocorticoid receptor-mediated control of genomic responses to stress in the brain. Stress 2018; 21(5): 389-402.

123. Hauger RL, Risbrough V, Brauns O, Dautzenberg FM. Corticotropin releasing factor (CRF) receptor signaling in the central nervous system: new molecular targets. CNS Neurol Disord Drug Targets 2006; 5(4): 453-479.

124. Grammatopoulos DK. Insights into mechanisms of corticotropin-releasing hormone receptor signal transduction. Br J Pharmacol 2012; 166(1): 85-97. 


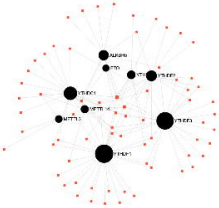




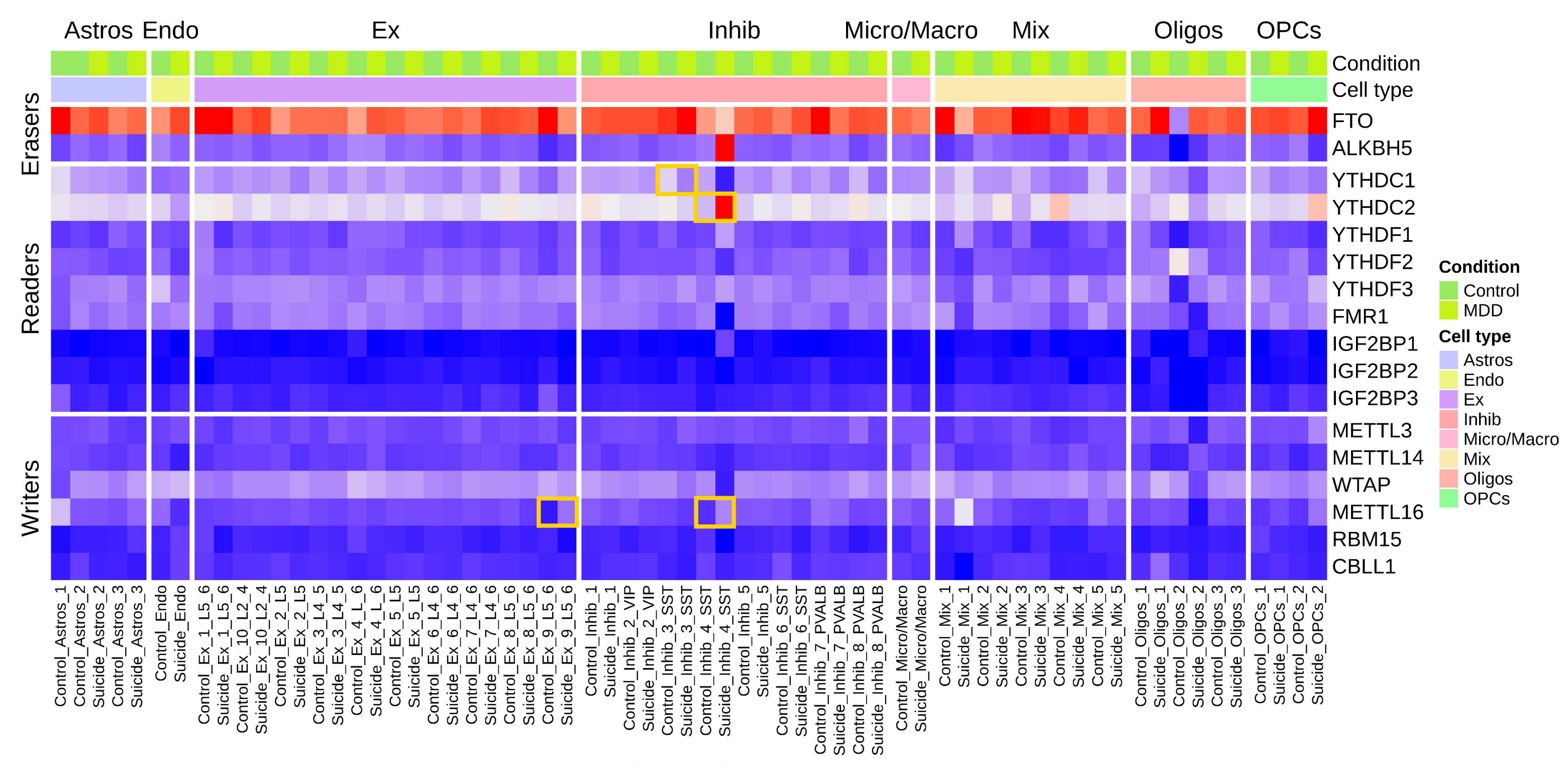

Mean log-normalised expression 


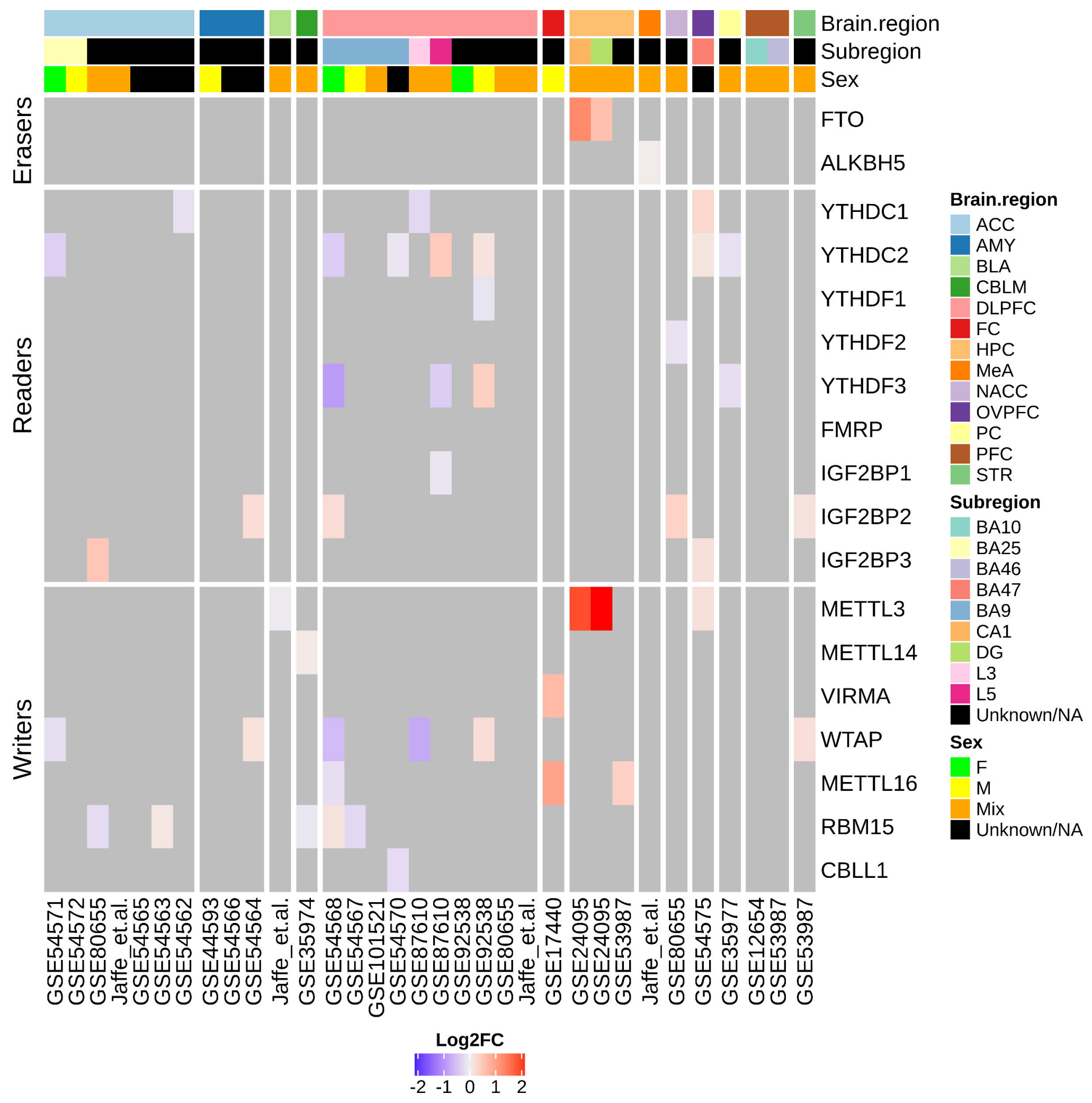




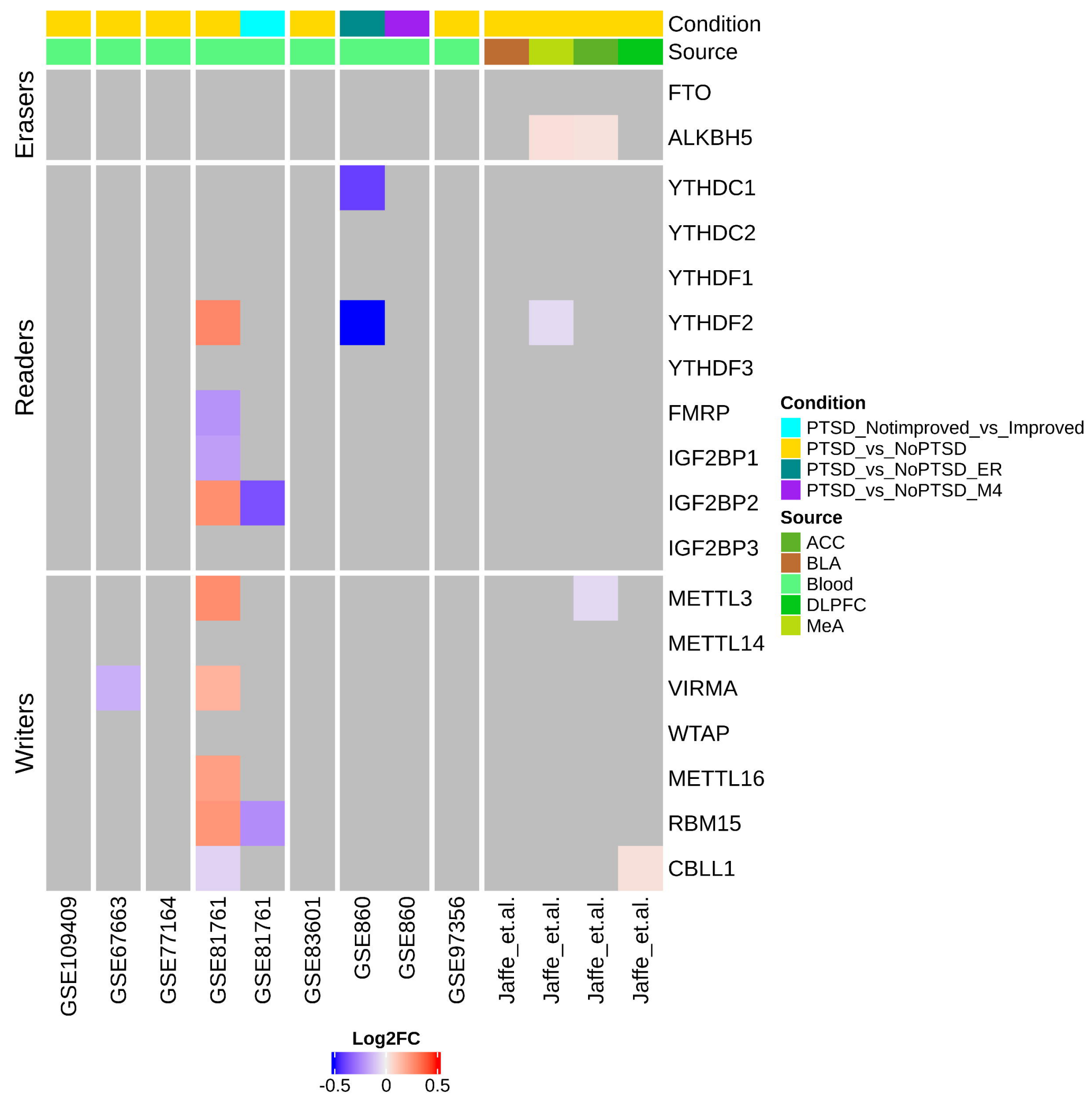




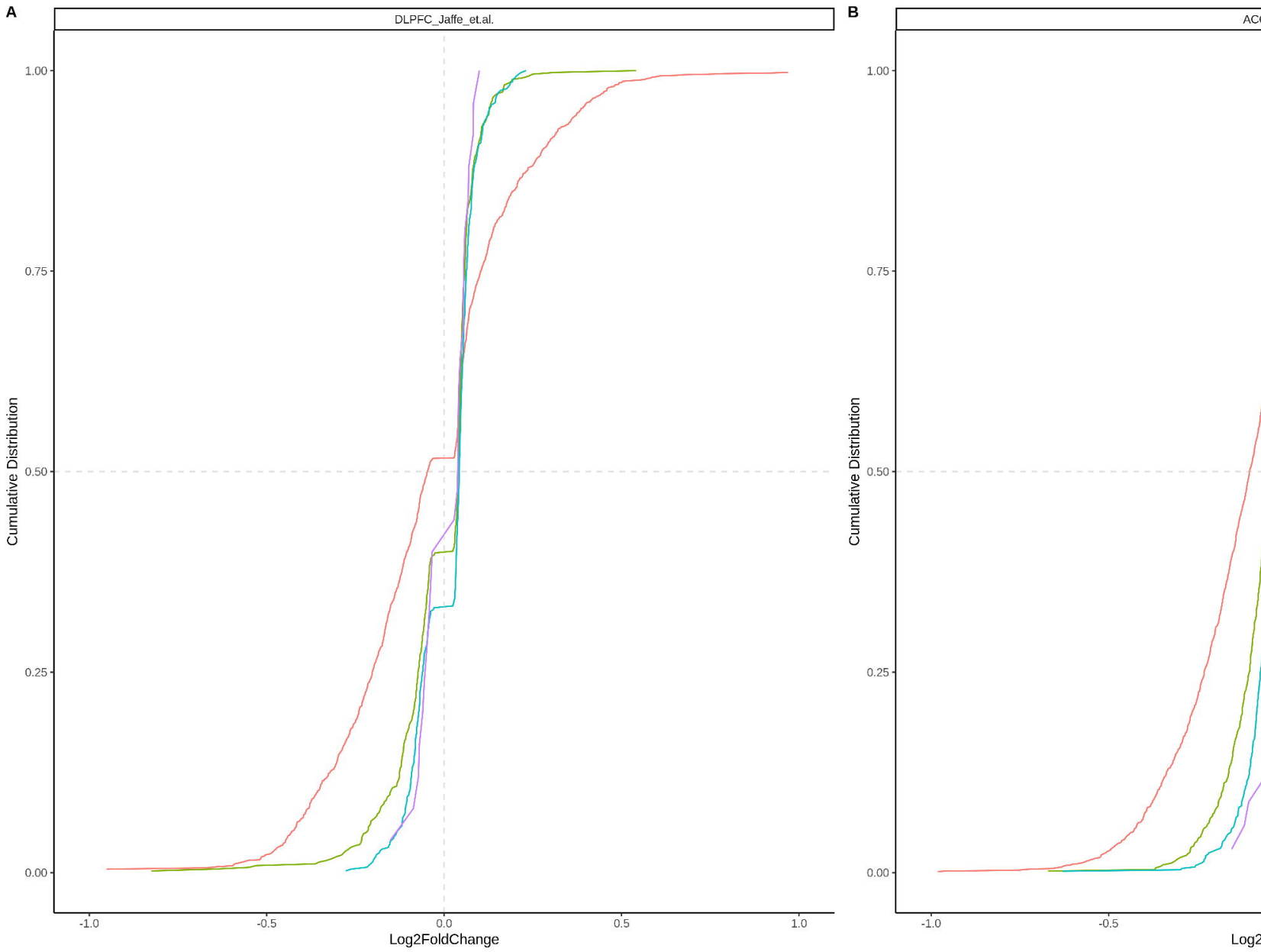



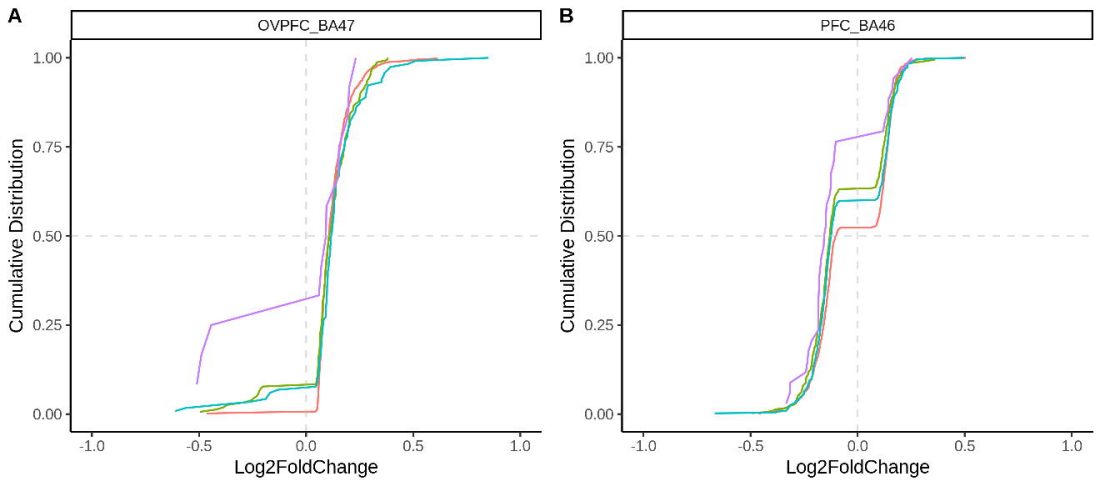

C

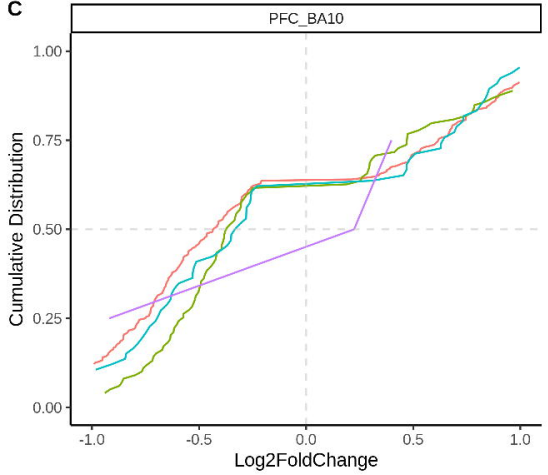

E

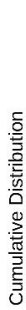

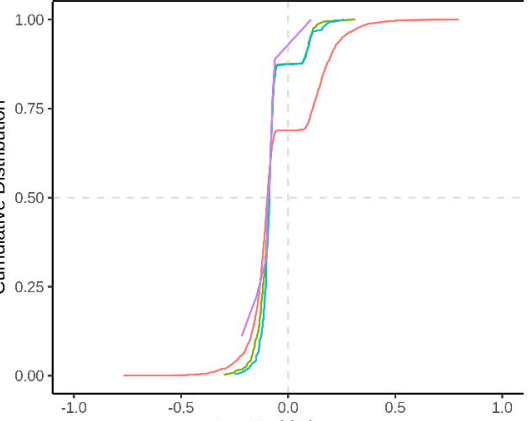

D

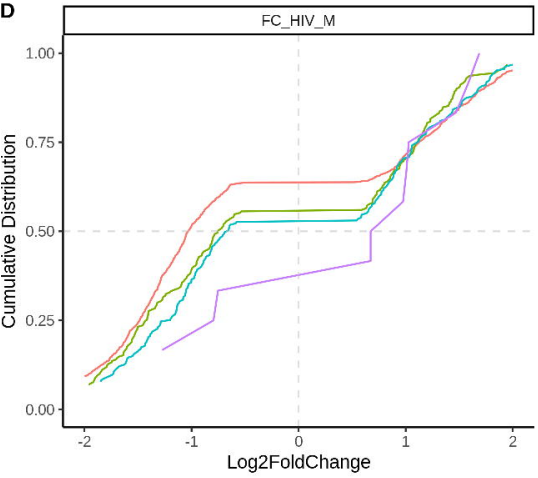

m6a_counts

- m6a_o

- m6a 1

- m6a_2 5

- m6a 6 